\title{
The Topp-Leone Marshall-Olkin-G Family of Distributions With Applications
}

\author{
Fastel Chipepa $^{1,2}$, Broderick Oluyede ${ }^{1} \&$ Boikanyo Makubate $^{1}$ \\ ${ }^{1}$ Department of Mathematics and Statistical Sciences, Botswana International University of Science and Technology, P. \\ Bag 16, Palapye, Botswana \\ 2 Department of Applied Mathematics and Statistics, Midlands State University, P. Bag 9055, Gweru, Zimbabwe \\ Correspondence: Department of Mathematics and Statistical Sciences, Botswana International University of Science and \\ Technology, P. Bag 16, Palapye, Botswana. E-mail: oluyedeo@biust.ac.bw
}

Received: April 15, 2020 Accepted: May 13, 2020 Online Published: May 15, 2020

doi:10.5539/ijsp.v9n4p15 URL: https://doi.org/10.5539/ijsp.v9n4p15

\begin{abstract}
A new generalized distribution is developed, namely, Topp-Leone Marshall-Olkin-G distribution. The new distribution is a linear combination of the exponentiated-G family of distributions. We considered three sub-families of the new proposed family of distribution. The distribution can handle heavy-tailed data and various forms of the hazard rate functions. A simulation study was conducted to evaluate consistency of the model parameters. Three applications are provided to demonstrate the usefulness of the new model in comparison with competing non-nested models.
\end{abstract}

Keywords: Topp-Leone distribution, Marshall-Olkin-G, maximum likelihood estimation

\section{Introduction}

There is an increase in the demand for generalized distributions, which can handle various levels of skewness and kurtosis. More so, there is an increased need for generalized distributions that can fit data that exhibit various shapes for the hazard rate functions. These generalized models has wider applications in areas of reliability and engineering. Also, these generalized models have wider applications in hydrology, medicine, economics, finance and insurance. In response to this demand, many generators are proposed in literature and these include beta-G by Eugene, Lee, and Famoye (2002), Marshall-Olkin-G (MO-G) by Marshall and Olkin (1997), Kumaraswamy-G (Kw-G) by Cordeiro and de Castro (2011), gamma-G by Zografos and Balakrishinan (2009), Weibull-G (W-G) by Bourguignon, Silva, and Cordeiro (2014), T-X family by Alzaatreh and Ghosh (2013), beta odd Lindley-G (BOL-G) by Chipepa, Oluyede, Makubate, and Fagbamigbe (2019), Kumaraswamy odd Lindley-G by Chipepa, Oluyede, and Makubate (2019), Topp-Leone odd log-logistic-G (TLOLL-G) by Brito, Cordeiro, Yousof, Alizadeh, and Silva (2017), to mention a few.

Furthermore, Topp and Leone (1955) developed a model that always exhibits the bathtub shaped hazard rates. The ToppLeone distribution is an extension of the triangular distribution, and as such it is not a very flexible distribution since its domain is restricted to $(0,1)$. This distribution has cumulative distribution function (cdf) defined as

$$
F_{T L}(x)=\left[1-(1-x)^{2}\right]^{b},
$$

for $0<x<1$ and $b>0$.

Marshall and Olkin (1997), introduced a new distribution defined by

$$
F_{M O}(x)=1-\frac{\delta \bar{G}(x)}{1-\bar{\delta} \bar{G}(x)},
$$

where $\delta$ is the tilt parameter and $G(x)$ is the baseline cdf. The MO-G distribution is more flexible compared to other distributions like exponential, Weibull and gamma. We propose a new family that enables us to model data that are

- heavy tailed

- heavily skewed

- platykurtic and leptokurtic compared to the baseline distribution 
- The proposed new model can be applied to data that have non-monotonic hazard rate functions.

In this paper, we develop the Topp-Leone Marshall-Olkin-G (TL-MO-G) family of distributions. In Section 2, we develop the new generalized distribution. Section 3 contains sub-families. In Section 4, we presents structural properties of the new distribution. In Section 5, we derive maximum likelihood estimates. We present results of the simulation study in Section 6. In Section 7, we present applications of the new model to real data examples, followed by concluding remarks.

\section{The Topp-Leone-Marshall-Olkin-G Family of Distributions}

We use Equation (1), and the generalization by Marshall and Olkin given in Equation (2) to derive the Topp-LeoneMarshall-Olkin-G (TL-MO-G) family of distributions. Therefore, the TL-MO-G family of distributions is given by

$$
F_{T L-M O-G}(x ; b, \delta, \xi)=\left[1-\bar{G}_{M O}^{2}(x ; \xi)\right]^{b}=\left[1-\frac{\delta^{2} \bar{G}^{2}(x ; \xi)}{[1-\bar{\delta} \bar{G}(x ; \xi)]^{2}}\right]^{b},
$$

with corresponding probability density function (pdf)

$$
\begin{aligned}
f_{T L-M O-G}(x ; b, \delta, \xi) & =\left[\frac{2 b \delta g(x ; \xi)}{[1-\bar{\delta} \bar{G}(x ; \xi)]^{2}}\right]\left[\frac{\delta \bar{G}(x ; \xi)}{[1-\bar{\delta} \bar{G}(x ; \xi)]}\right]\left[1-\frac{\delta^{2} \bar{G}^{2}(x ; \xi)}{[1-\bar{\delta} \bar{G}(x ; \xi)]^{2}}\right]^{b-1} \\
& =\left[\frac{2 b \delta^{2} g(x ; \xi) \bar{G}(x ; \xi)}{[1-\bar{\delta} \bar{G}(x ; \xi)]^{3}}\right]\left[1-\frac{\delta^{2} \bar{G}^{2}(x ; \xi)}{[1-\bar{\delta} \bar{G}(x ; \xi)]^{2}}\right]^{b-1}
\end{aligned}
$$

for $b, \delta>0, \bar{\delta}=1-\delta$ and $\xi$ is a vector of parameters from the baseline distribution $\mathrm{G}($.$) .$

\subsection{Linear Representation}

We derive the series representation of the TL-MO-G distribution using the pdf of the TL-MO-G distribution and the series expansion

$$
\left[1-\frac{\delta^{2} \bar{G}^{2}(x ; \xi)}{[1-\bar{\delta} \bar{G}(x ; \xi)]^{2}}\right]^{b-1}=\sum_{n=0}^{\infty} \frac{(-1)^{n} \Gamma(b) \delta^{2 n} \bar{G}^{2 n}(x ; \xi)}{\Gamma(b-n) n ![1-\bar{\delta} \bar{G}(x ; \xi)]^{2 n}}
$$

we have

$$
f(x ; b, \delta, \xi)=2 b \sum_{n=0}^{\infty} \frac{(-1)^{n} \Gamma(b) \delta^{2 n+2} g(x ; \xi) \bar{G}^{2 n+1}(x ; \xi)}{\Gamma(b-n) n ![1-\bar{\delta} \bar{G}(x ; \xi)]^{2 n+3}}
$$

and applying the following binomial expansion

$$
[1-\bar{\delta} \bar{G}(x ; \xi)]^{-(2 n+3)}=\sum_{j=0}^{\infty}\left(\begin{array}{c}
-(2 n+3) \\
j
\end{array}\right) \bar{\delta}^{j} \bar{G}^{j}(x ; \xi)
$$

we have

$$
\begin{aligned}
f(x ; b, \delta, \xi) & =2 b \sum_{j, n=0}^{\infty} \frac{(-1)^{n} \Gamma(b) \delta^{2 n+2} \bar{\delta}^{j}}{\Gamma(b-n) n !}\left(\begin{array}{c}
-(2 n+3) \\
j
\end{array}\right) \\
& \times g(x ; \xi) \bar{G}^{2 n+j+1}(x ; \xi) .
\end{aligned}
$$

By applying the binomial expansion

$$
\bar{G}^{2 n+j+1}(x ; \xi)=[1-G(x ; \xi)]^{2 n+j+1}=\sum_{p=0}^{\infty}(-1)^{p}\left(\begin{array}{c}
2 n+j+1 \\
p
\end{array}\right) G^{p}(x ; \xi),
$$

we can therefore, write the linear representation of the TL-MO-G distribution as

$$
\begin{aligned}
f(x ; b, \delta, \xi) & =2 b \sum_{p, j, n=0}^{\infty} \frac{(-1)^{n+p} \Gamma(b) \delta^{2 n+2} \bar{\delta}^{j}}{(p+1) \Gamma(b-n) n !}\left(\begin{array}{c}
-(2 n+3) \\
j
\end{array}\right)\left(\begin{array}{c}
2 n+j+1 \\
p
\end{array}\right) \\
& \times(p+1) g(x ; \xi) G^{p}(x ; \xi) \\
& =\sum_{p=0}^{\infty} v_{p} g_{p}(x ; \xi) .
\end{aligned}
$$


where

$$
v_{p}=2 b \sum_{j, n=0}^{\infty} \frac{(-1)^{n+p} \Gamma(b) \delta^{2 n+2} \bar{\delta}^{j}}{(p+1) \Gamma(b-n) n !}\left(\begin{array}{c}
-(2 n+3) \\
j
\end{array}\right)\left(\begin{array}{c}
2 n+j+1 \\
p
\end{array}\right)
$$

and $g_{p}(x ; \xi)=(p+1) g(x ; \xi) G^{p}(x ; \xi)$ is an exponentiated-G (Exp-G) distribution with parameter $p$. The TL-MO-G family of distributions is a family of the Exp-G distributions.

\section{Sub-Families}

We present some sub-families of TL-MO-G distribution. We considered cases when the baseline distributions are uniform, log-logistic, Weibull and normal distributions.

\subsection{Topp-Leone-Marshall-Olkin-Uniform (TL-MO-U) Distribution}

By taking the baseline distribution to be uniform distribution, we obtain the Topp-Leone-Marshall-Olkin-Uniform (TLMO-U) distribution. The uniform distribution has $g(x)=1 / \theta$ and $G(x, \theta)=x / \theta$, for $0<x<\theta$. Therefore, the TL-MO-U distribution is given by

$$
F_{T L-M O-U}(x ; b, \delta, \theta)=\left[1-\frac{\delta^{2}[1-x / \theta]^{2}}{[1-\bar{\delta}(1-x / \theta)]^{2}}\right]^{b}
$$

with pdf

$$
f_{T L-M O-U}(x ; b, \delta, \theta)=\frac{2 b \delta^{2} x(1-x / \theta)}{\theta[1-\bar{\delta}(1-x / \theta)]^{3}}\left[1-\frac{\delta^{2}[1-x / \theta]^{2}}{[1-\bar{\delta}(1-x / \theta)]^{2}}\right]^{b-1}
$$

for $b, \delta$ and $\theta>0$.
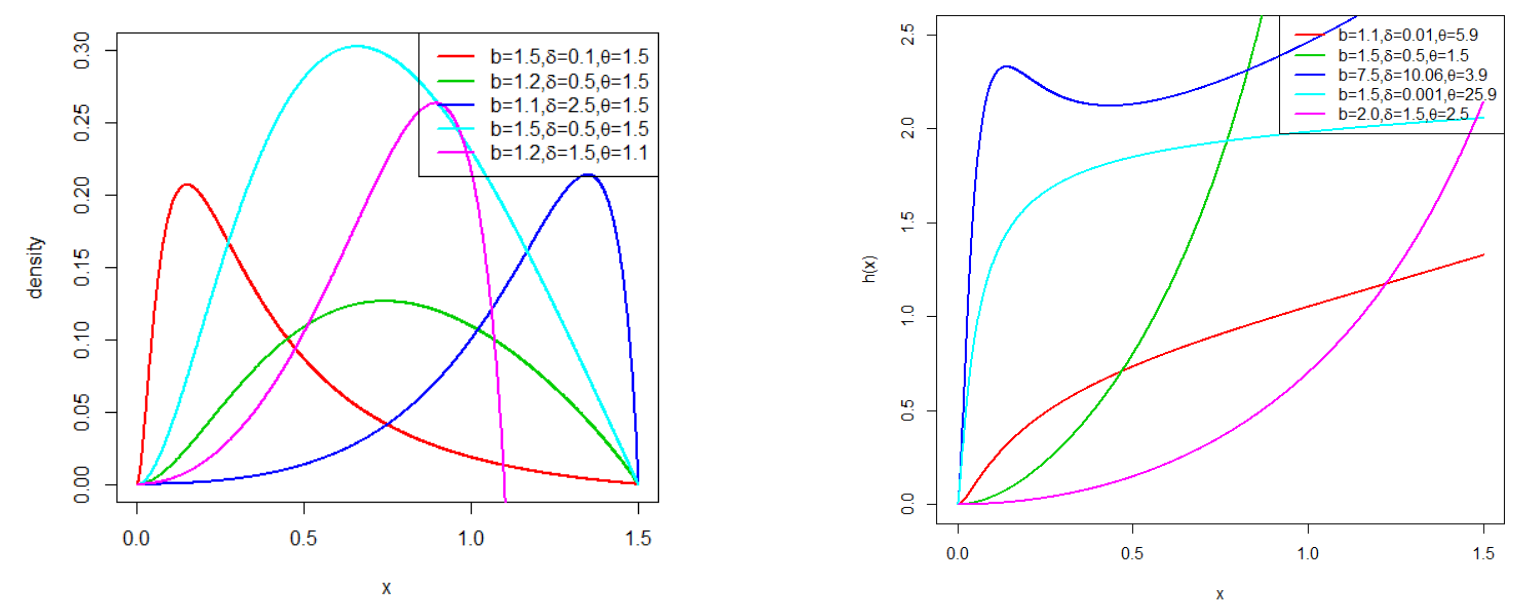

Figure 1. Plots of the pdf and hrf for the TL-MO-U distribution

From Figures 1 we deduce that the new distribution can handle data that is symmetric, left or right skewed and that the TL-MO-U model can fit data sets that have an increasing, upside bathtub followed by bathtub hazard rate functions (hrf).

\subsection{Topp-Leone-Marshall-Olkin-Log-Logistic (TL-MO-LLo) Distribution}

By taking the baseline distribution to be the log-logistic distribution we obtain the Topp-Leone-Marshall-Olkin-loglogistic (TL-MO-LLo) distribution. The log-logistic distribution has $g(x)=c x^{c-1}\left(1+x^{c}\right)^{-2}$ and $G(x)=1-\left(1+x^{c}\right)^{-1}$, for $c>0$, respectively. Therefore, the TL-MO-LLo distribution is given by

$$
F_{T L-M O-L L O}(x ; b, \delta, c)=\left[1-\frac{\delta^{2}\left[1+x^{c}\right]^{-2}}{\left[1-\bar{\delta}\left(1+x^{c}\right)^{-1}\right]^{2}}\right]^{b},
$$

with pdf

$$
f_{T L-M O-L L o}(x ; b, \delta, c)=\frac{2 b \delta^{2} c x^{c-1}\left(1+x^{c}\right)^{-3}}{\left[1-\bar{\delta}\left(1+x^{c}\right)^{-1}\right]^{3}}\left[1-\frac{\delta^{2}\left[1+x^{c}\right]^{-2}}{\left[1-\bar{\delta}\left(1+x^{c}\right)^{-1}\right]^{2}}\right]^{b-1},
$$



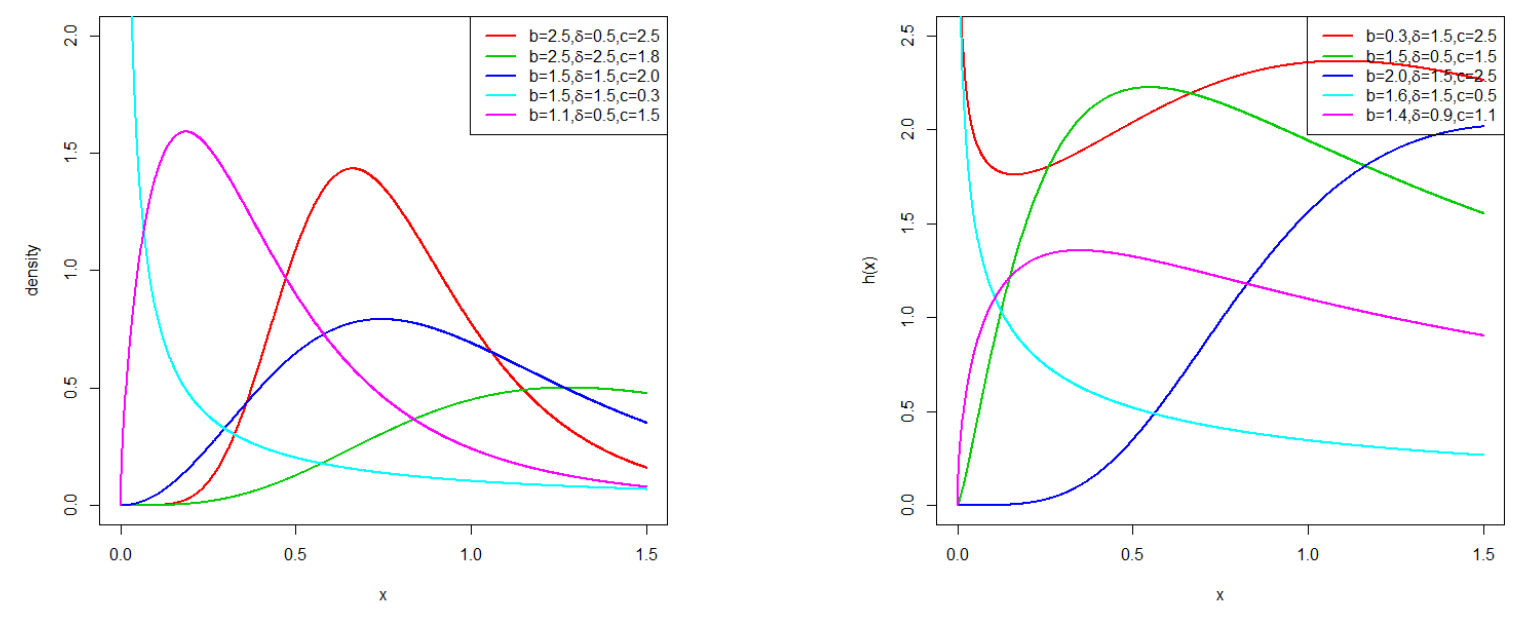

Figure 2. Plots of the pdf and hrf for the TL-MO-LLo distribution

respectively, for $b, \delta, c>0$.

Figure 2 show that the new distribution applies to heavy-tailed data. The distribution also addresses the variation in both kurtosis and skewness. The TL-MO-LLo model can fit to various hazard rates that includes bathtub followed by upside down bathtub, decreasing, increasing, and upside bathtub or uni-modal.

\subsection{The Topp-Leone Marshall-Olkin-Weibull Distribution}

By taking the basine distribution to be Weibull distribution, we obtain the Topp-Leone-Marshall-Olkin-Weibull (TLMO-W)distribution. Weibull distribution has $g(x ; \lambda, \omega)=\lambda \omega x^{\omega-1} e^{-\lambda x^{\omega}}$ and $G(x ; \lambda, \omega)=1-e^{-\lambda x^{\omega}}$, for $\lambda, \omega>0$. The TL-MO-W distribution is given by

$$
F_{T L-M O-W}(x ; b, \delta, \lambda, \gamma)=\left[1-\frac{\delta^{2} e^{-2 \lambda x^{\gamma}}}{\left(1-\bar{\delta} e^{-\lambda x^{\gamma}}\right)^{2}}\right]^{b},
$$

with pdf

$$
f_{T L-M O-W}(x ; b, \delta, \lambda, \gamma)=\frac{2 b \delta^{2} \lambda \gamma x^{\gamma-1} e^{-2 \lambda x^{\gamma}}}{\left(1-\bar{\delta} e^{-\lambda x^{\gamma}}\right)^{3}}\left[1-\frac{\delta^{2} e^{-2 \lambda x^{\gamma}}}{\left(1-\bar{\delta} e^{-\lambda x^{\gamma}}\right)^{2}}\right]^{b-1}
$$

for $b, \delta, \lambda, \omega>0$.

The pdfs of the TL-MO-W distribution can take uni-modal, left or right skewed and reverse-J shapes. Also, the TLMO-W distribution exhibits various shapes for the hazard rate function.

\subsection{The Topp-Leone Marshall-Olkin-Normal Distribution}

Consider the normal distribution with pdf $g(x ; \mu, \sigma)=\sigma^{-1} \phi\left(\frac{x-\mu}{\sigma}\right)$ and $\operatorname{cdf} G(x ; \mu, \sigma)=\Phi\left(\frac{x-\mu}{\sigma}\right)$, for $\mu \in \mathfrak{R}$ and $\sigma>0$, as the baseline distribution, we obtain the Topp-Leone Marshall-Olkin-normal (TL-MO-N) distribution with cdf and pdf given by

$$
F_{T L-M O-N}(x ; b, \delta, \mu, \sigma)=\left[1-\frac{\delta^{2}\left(1-\Phi\left(\frac{x-\mu}{\sigma}\right)\right)^{2}}{\left(1-\bar{\delta}\left(1-\Phi\left(\frac{x-\mu}{\sigma}\right)\right)\right)^{2}}\right]^{b}
$$

and

$$
f_{T L-M O-N}(x ; b, \delta, \mu, \sigma)=\frac{2 b \delta \phi\left(\frac{x-\mu}{\sigma}\right)\left[\left(1-\Phi\left(\frac{x-\mu}{\sigma}\right)\right)\right]}{\sigma\left[1-\bar{\delta}\left(1-\Phi\left(\frac{x-\mu}{\sigma}\right)\right)\right]^{3}}\left[1-\frac{\delta^{2}\left(1-\Phi\left(\frac{x-\mu}{\sigma}\right)\right)^{2}}{\left(1-\bar{\delta}\left(1-\Phi\left(\frac{x-\mu}{\sigma}\right)\right)\right)^{2}}\right]^{b-1},
$$

for $b, \delta, \sigma>0$ and $-\infty<\mu<\infty$. 

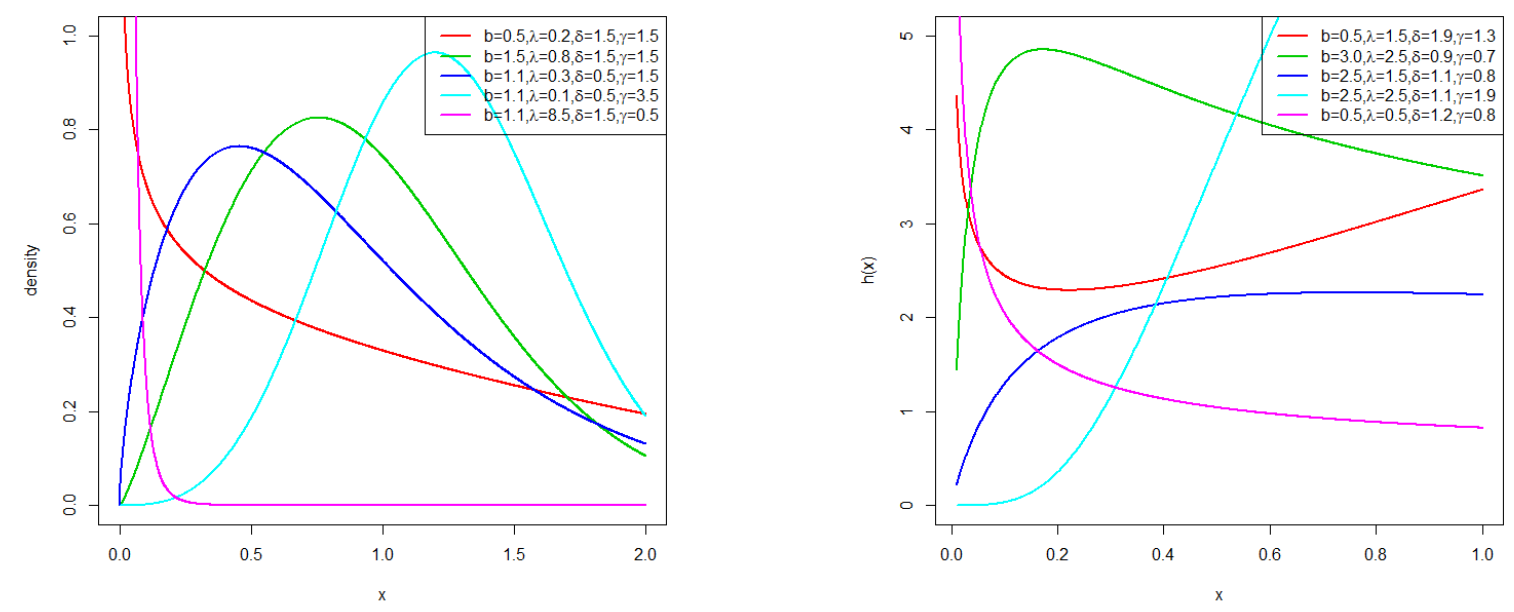

Figure 3. Plots of the pdf and hrf for the TL-MO-W distribution
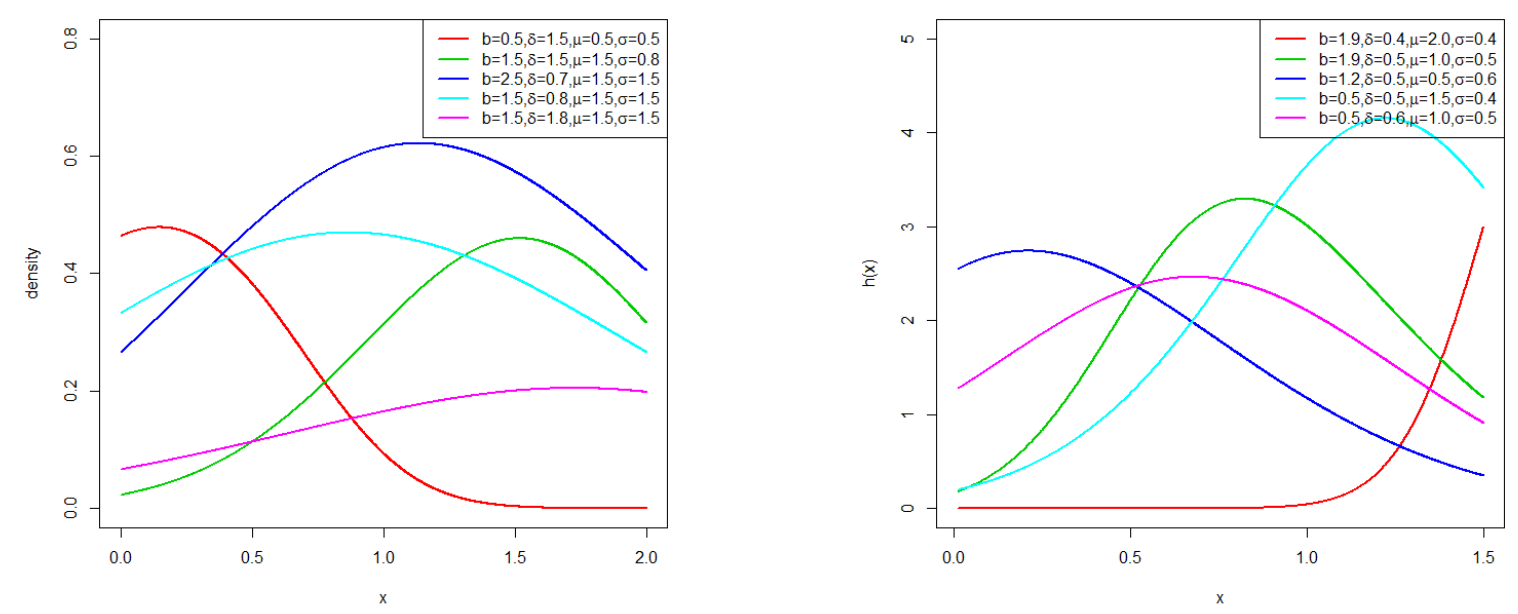

Figure 4. Plots of the pdf and hrf for the TL-MO-N distribution

Figure 4 show that the TL-MO-N distribution can take various shapes for its pdf. Also, the hazard rate function for the TL-MO-N distribution exhibit various shapes.

\section{Statistical Properties}

\subsection{Distribution of Order Statistics}

We can use equation (7) to determine the distribution of the $i^{\text {th }}$ order statistics from the TL-MO-G family of distributions.

$$
f_{i: n}(x)=\frac{f(x)}{B(i, n-i+1)} \sum_{j=0}^{n-j}\left(\begin{array}{c}
n-i \\
j
\end{array}\right) F(x)^{j+i-1},
$$

where $B(.,$.$) is the beta function. Using equations (3) and (4), f(x) F(x)^{j+i-1}$ from equation (7) simplifies to

$$
f(x) F(x)^{j+i-1}=\left[\frac{2 b \delta^{2} g(x ; \xi) \bar{G}(x ; \xi)}{[1-\bar{\delta} \bar{G}(x ; \xi)]^{3}}\right]\left[1-\frac{\delta^{2} \bar{G}^{2}(x ; \xi)}{[1-\bar{\delta} \bar{G}(x ; \xi)]^{2}}\right]^{b(j+i)-1},
$$

and by applying the binomial expansion

$$
\left[1-\frac{\delta^{2} \bar{G}^{2}(x ; \xi)}{[1-\bar{\delta} \bar{G}(x ; \xi)]^{2}}\right]^{b(j+i)-1}=\sum_{k=0}^{\infty} \frac{(-1)^{k} \Gamma(b(j+i)) \delta^{2 k} \bar{G}^{2 k}(x ; \xi)}{\Gamma(b(j+i)-k) k ![1-\bar{\delta} \bar{G}(x ; \xi)]^{2 k}}
$$


yields

$$
f(x) F(x)^{j+i-1}=2 b \sum_{k=0}^{\infty} \frac{(-1)^{k} \delta^{2 k+2} \Gamma(b(j+i)) g(x ; \xi) \bar{G}^{2 k+1}(x ; \xi)}{\Gamma(b(j+i)-k) k ![1-\bar{\delta} \bar{G}(x ; \xi)]^{2 k+3}} .
$$

Furthermore, applying the binomial expansion

$$
[1-\bar{\delta} \bar{G}(x ; \xi)]^{-(2 k+3)}=\sum_{m=0}^{\infty}\left(\begin{array}{c}
-(2 k+3) \\
m
\end{array}\right) \bar{\delta}^{m} \bar{G}^{m}(x ; \xi),
$$

we can write

$$
\begin{aligned}
f(x) F(x)^{j+i-1} & =2 b \sum_{k, m=0}^{\infty}(-1)^{k} \delta^{2 k+2} \bar{\delta}^{m}\left(\begin{array}{c}
-(2 k+3) \\
m
\end{array}\right) \frac{\Gamma(b(j+i))}{\Gamma(b(j+i)-k) k !} \\
& \times g(x ; \xi) \bar{G}^{m+2 k+1}(x ; \xi) .
\end{aligned}
$$

Also, applying the following binomial expansion

$$
\bar{G}^{m+2 k+1}(x ; \xi)=[1-G(x ; \xi)]^{m+2 k+1}=\sum_{q=0}^{\infty}(-1)^{q}\left(\begin{array}{c}
m+2 k+1 \\
q
\end{array}\right) G^{q}(x ; \xi),
$$

yields

$$
\begin{aligned}
f(x) F(x)^{j+i-1} & =2 b \sum_{q, k, m=0}^{\infty}(-1)^{k+q} \delta^{2 k+2} \bar{\delta}^{m}\left(\begin{array}{c}
-(2 k+3) \\
m
\end{array}\right)\left(\begin{array}{c}
m+2 k+1 \\
q
\end{array}\right) \\
& \times \frac{\Gamma(b(j+i))}{\Gamma(b(j+i)-k) k !} g(x ; \xi) G^{q}(x ; \xi) .
\end{aligned}
$$

Therefore,

$$
\begin{aligned}
f_{i: n}(x) & =2 b \sum_{q, k, m=0}^{\infty} \sum_{j=0}^{n-j} \frac{(-1)^{k+q} \delta^{2 k+2} \bar{\delta}^{m}}{B(i, n-i+1)}\left(\begin{array}{c}
n-i \\
j
\end{array}\right)\left(\begin{array}{c}
-(2 k+3) \\
m
\end{array}\right)\left(\begin{array}{c}
m+2 k+1 \\
q
\end{array}\right) \\
& \times \frac{\Gamma(b(j+i))}{(q+1) \Gamma(b(j+i)-k) k !}(q+1) g(x ; \xi) G^{q}(x ; \xi) \\
& =\sum_{q=0}^{\infty} v_{q}^{*} g_{q}(x ; \xi),
\end{aligned}
$$

where

$$
\begin{aligned}
v_{q}^{*} & =2 b \sum_{k, m=0}^{\infty} \sum_{j=0}^{n-j} \frac{(-1)^{k+q} \delta^{2 k+2} \bar{\delta}^{m}}{B(i, n-i+1)}\left(\begin{array}{c}
n-i \\
j
\end{array}\right)\left(\begin{array}{c}
-(2 k+3) \\
m
\end{array}\right)\left(\begin{array}{c}
m+2 k+1 \\
q
\end{array}\right) \\
& \times \frac{\Gamma(b(j+i))}{(q+1) \Gamma(b(j+i)-k) k !}
\end{aligned}
$$

and $g_{q}(x ; \xi)=(q+1) g(x ; \xi) G^{q}(x ; \xi)$ is the Exp-G distribution with parameter $q$. It follows that the $i^{t h}$ order statistics from the TL-MO-G distribution can be obtained directly from that of the Exp-G distribution.

\subsection{Entropy}

There are two common measures of entropy and these are Rényi entropy by Rényi (1960) and Shannon entropy by Shannon (1951). In this paper, we derive the Rényi entropy $\left(I_{R}(v)\right)$ of the TL-MO-G family of distributions using the formula

$$
I_{R}(v)=(1-v)^{-1} \log \left[\int_{0}^{\infty} f^{v}(x) d x\right], v \neq 1, v>0 .
$$

Substituting Equation (4) for $f(x)$, we get

$$
f^{v}(x)=\frac{(2 b)^{v} \delta^{2 v} g^{v}(x ; \xi) \bar{G}^{v}(x ; \xi)}{[1-\bar{\delta} \bar{G}(x ; \xi)]^{3 v}}\left[1-\frac{\delta^{2} \bar{G}^{2}(x ; \xi)}{[1-\bar{\delta} \bar{G}(x ; \xi)]^{2}}\right]^{v(b-1)} .
$$


Applying the binomial expansion

$$
\left[1-\frac{\delta^{2} \bar{G}^{2}(x ; \xi)}{[1-\bar{\delta} \bar{G}(x ; \xi)]^{2}}\right]^{v(b-1)}=\sum_{j=0}^{\infty}(-1)^{j} \frac{\Gamma(v(b-1)+1)}{\Gamma(v(b-1)+1-j) j !} \frac{\delta^{2 j} \bar{G}^{2 j}(x ; \xi)}{[1-\bar{\delta} \bar{G}(x ; \xi)]^{2 j}},
$$

we can write

$$
f^{v}(x)=\sum_{j=0}^{\infty}(-1)^{j}(2 b)^{v} \delta^{2(v+j)} \frac{\Gamma(v(b-1)+1)}{\Gamma(v(b-1)+1-j) j !} \frac{g^{v}(x ; \xi) \bar{G}^{v+2 j}(x ; \xi)}{[1-\bar{\delta} \bar{G}(x ; \xi)]^{3 v+2 j}} .
$$

Also, considering the binomial expansion

$$
[1-\bar{\delta} \bar{G}(x ; \xi)]^{-(3 v+2 j)}=\sum_{m=0}^{\infty}\left(\begin{array}{c}
-(3 v+2 j) \\
m
\end{array}\right) \bar{\delta}^{m} \bar{G}^{m}(x ; \xi)
$$

yields

$$
\begin{aligned}
f^{v}(x) & =\sum_{j, m=0}^{\infty}(-1)^{j}(2 b)^{v} \delta^{2(v+j)+m} \frac{\Gamma(v(b-1)+1)}{\Gamma(v(b-1)+1-j) j !}\left(\begin{array}{c}
-(3 v+2 j) \\
m
\end{array}\right) \\
& \times g^{v}(x ; \xi)[\bar{G}(x ; \xi)]^{v+2 j+m} .
\end{aligned}
$$

Also, applying the following binomial expansion

$$
\bar{G}^{v+2 j+m}(x ; \xi)=[1-G(x ; \xi)]^{v+2 j+m}=\sum_{l=0}^{\infty}(-1)^{l}\left(\begin{array}{c}
v+2 j+m \\
l
\end{array}\right) G^{l}(x ; \xi),
$$

we can therefore, write the Rényi entropy of the TL-MO-G family of distributions as

$$
\begin{aligned}
f^{v}(x) & =(1-v)^{-1} \log \left[\sum_{l, j, m=0}^{\infty}(-1)^{j+l}(2 b)^{v} \delta^{2(v+j)+m} \frac{\Gamma(v(b-1)+1)}{\Gamma(v(b-1)+1-j) j !}\right. \\
& \times\left(\begin{array}{c}
-(3 v+2 j) \\
m
\end{array}\right)\left(\begin{array}{c}
v+2 j+m \\
l
\end{array}\right)\left(\frac{1}{\frac{l}{v}+1}\right)^{v} \\
& \left.\times \int_{0}^{\infty}\left[\left(\frac{l}{v}+1\right) g(x ; \xi) G^{l / v}(x ; \xi)\right]^{v} d x\right] \\
& =(1-v)^{-1} \log \left[\sum_{l=0}^{\infty} w_{l}^{*} e^{(1-v) I_{R E G}}\right],
\end{aligned}
$$

where

$$
\begin{aligned}
w_{l}^{*} & =\sum_{j, m=0}^{\infty}(-1)^{j+l}(2 b)^{v} \delta^{2(v+j)+m} \frac{\Gamma(v(b-1)+1)}{\Gamma(v(b-1)+1-j) j !}\left(\begin{array}{c}
-(3 v+2 j) \\
m
\end{array}\right) \\
& \times\left(\begin{array}{c}
v+2 j+m \\
l
\end{array}\right)\left(\frac{1}{\frac{l}{v}+1}\right)^{v}
\end{aligned}
$$

and $I_{R E G}=\int_{0}^{\infty}\left[\left(\frac{l}{v}+1\right) g(x ; \xi) G^{l / v}(x ; \xi)\right]^{v} d x$ is Rényi entropy of Exp-G distribution with parameter $\frac{l}{v}$. It follows that the Rényi entropy of the TL-MO-G distribution can be obtained directly from the Exp-G distribution.

\subsection{Moments and Moment Generating Function}

We derive the $s^{\text {th }}$ ordinary moment of the TL-MO-G family of distributions using Equation (5) and is given by

$$
\mu_{s}^{\prime}=E\left(X^{s}\right)=\sum_{p=0}^{\infty} v_{p} E\left(Y_{p}\right)
$$

where $Y_{p}$ is an Exp-G distribution with power parameter $p$ and $v_{p}$ is given by Equation (6). The $r^{\text {th }}$ central moment of $X$ is given by

$$
\mu_{r}=\sum_{s=0}^{r}\left(\begin{array}{l}
r \\
s
\end{array}\right)\left(-\mu_{1}^{\prime}\right)^{r-s} E\left(X^{s}\right)=\sum_{s=0}^{r} \sum_{p=0}^{\infty} v_{p}\left(\begin{array}{l}
r \\
s
\end{array}\right)\left(-\mu_{1}^{\prime}\right)^{r-s} E\left(Y_{p}\right) .
$$


The cumulants of $X$ follow recursively from

$$
k_{r}=\mu_{r}^{\prime}-\sum_{s=0}^{r-1}\left(\begin{array}{l}
r-1 \\
s-1
\end{array}\right) k_{s} \mu_{r-s}^{\prime},
$$

where $k_{1}=\mu_{1}^{\prime}, k_{2}=\mu_{2}^{\prime}-\mu_{1}^{\prime 2}, k_{3}=\mu_{3}^{\prime}-3 \mu_{2}^{\prime} \mu_{1}^{\prime}+\mu_{1}^{\prime 3}$, etc. We use ordinary moments to determine the measures of spread, which includes, standard deviation, kurtosis and skewness.

Furthermore, we can find the $r^{\text {th }}$ incomplete moment of $X$ as follows

$$
\phi_{r}(z)=\int_{-\infty}^{z} x^{r} f(x) d x=\sum_{p=0}^{\infty} v_{p} \int_{-\infty}^{z} x^{r} g_{p}(x ; \xi) d x .
$$

We use the incomplete moment to estimate Lorenz and Bonferroni curves, which are useful in science, engineering, economics and demography. These quantities can be expressed mathematically by $L(p)=\phi_{1}(q) / \mu_{1}^{\prime}$ and $B(p)=\phi_{1}(q) /\left(p \mu_{1}^{\prime}\right)$, respectively, where $\mu_{1}^{\prime}$ is given by equation (14), with $r=1$ and $q=Q(p)$ is the quantile function of $X$ at $\mathrm{p}$. The incomplete moment (equation (15)) can also be expressed as

$$
\phi_{r}(z)=\sum_{p=0}^{\infty} v_{p} H_{p}(z)
$$

where $H_{p}(z)=\int_{-\infty}^{z} x^{r} g_{p}(x ; \xi) d x$ is the $r^{\text {th }}$ incomplete moment of the Exp-G distribution.

We present the first five moments of the TL-MO-LLo distribution, and the standard deviation (SD or $\sigma$ ), coefficient of variation $(\mathrm{CV})$, coefficient of skewness (CS) and coefficient of kurtosis (CK) for selected parameters values. The results are shown in Table 1.

Table 1. Moments of the TL-MO-LLo distribution

\begin{tabular}{cccccc}
\hline & $(0.9,1.5,1.5)$ & $(0.5,1,0.5)$ & $(1,0.5,1.5)$ & $(1.5,1.5,0.5)$ & $(0.5,0.1,0.5)$ \\
\hline$E(X)$ & 0.3079 & 0.0886 & 0.3184 & 0.1484 & 0.0113 \\
$E\left(X^{2}\right)$ & 0.1893 & 0.0413 & 0.1655 & 0.0820 & 0.0032 \\
$E\left(X^{3}\right)$ & 0.1338 & 0.0265 & 0.1049 & 0.0561 & 0.0017 \\
$E\left(X^{4}\right)$ & 0.1026 & 0.0195 & 0.0747 & 0.0425 & 0.0012 \\
$E\left(X^{5}\right)$ & 0.0828 & 0.0154 & 0.0573 & 0.0342 & 0.0009 \\
SD & 0.3074 & 0.1829 & 0.2532 & 0.2450 & 0.0551 \\
CV & 0.9986 & 2.0649 & 0.7952 & 1.6511 & 4.8932 \\
CS & 0.5982 & 2.7676 & 0.6998 & 1.7767 & 9.8177 \\
CK & 2.0668 & 10.5651 & 2.6679 & 5.1602 & 121.8401 \\
\hline
\end{tabular}

Furthermore, we obtain the moment generating function (mgf) of the TL-MO-G distribution

$$
M_{x}(t)=E\left(e^{t X}\right)=\sum_{p=0}^{\infty} v_{p} M_{p}(t),
$$

where $M_{p}(t)$ is the mgf of Exp-G distribution.

\subsection{Probability Weighted Moments}

We can use Probability Weighted Moments (PWMs) to estimate parameters of distributions which are not in closed form. The $(j, i)^{\text {th }} \mathrm{PWM}$, say $\eta_{j, i}$ of $X \sim \mathrm{TL}-\mathrm{MO}-\mathrm{G}(b, \delta ; \xi)$ distribution is defined by

$$
\eta_{j, i}=E\left(X^{j} F(X)^{i}\right)=\int_{-\infty}^{\infty} x^{j} f(x) F(x)^{i} d x .
$$

From equation (8), we have

$$
\begin{aligned}
f(x) F(x)^{i} & =2 b \sum_{q, k, m=0}^{\infty}(-1)^{k+q} \delta^{2 k+2} \bar{\delta}^{m}\left(\begin{array}{c}
-(2 k+3) \\
m
\end{array}\right)((m+2 k+1) \\
& \times g(x ; \xi) G^{q}(x ; \xi) .
\end{aligned}
$$


which simplifies to

$$
f(x) F(x)^{i}=\sum_{q=0}^{\infty} z_{q}^{*} g_{q}(x ; \xi)
$$

where

$$
z_{q}^{*}=2 b \sum_{q=0}^{\infty}(-1)^{k+q} \delta^{2 k+2} \bar{\delta}^{m}\left(\begin{array}{c}
-(2 k+3) \\
m
\end{array}\right)\left(\begin{array}{c}
(m+2 k+1) \\
\Gamma(b i-k) k !(q+1)
\end{array}\right.
$$

and $g_{q}(x ; \xi)$ is an Exp-G pdf. Therefore, the PWM is given by

$$
\begin{aligned}
\eta_{j, i} & =\sum_{q=0}^{\infty} z_{q}^{*} \int_{-\infty}^{\infty} x^{j} g_{q}(x ; \xi) d x \\
& =\sum_{q=0}^{\infty} z_{q}^{*} E\left(T_{q}^{j}\right),
\end{aligned}
$$

where $T_{q}^{j}$ is $j^{\text {th }}$ power of an Exp-G distributed random variable with power parameter $q$.

\subsection{Quantile Function}

To obtain the quantile function of the TL-MO-G distribution, we invert the cdf given in equation (3). Note that

$$
\left[1-\frac{\delta^{2} \bar{G}^{2}(x ; \xi)}{[1-\bar{\delta} \bar{G}(x ; \xi)]^{2}}\right]^{b}=u
$$

can be written as

$$
1-u^{1 / b}=\frac{\delta^{2} \bar{G}^{2}(x ; \xi)}{[1-\bar{\delta} \bar{G}(x ; \xi)]^{2}}
$$

which reduces to

$$
\left[\frac{1-u^{1 / b}}{\delta^{2}}\right]^{1 / 2}=\frac{\bar{G}(x ; \xi)}{1-\bar{\delta} \bar{G}(x ; \xi)}
$$

which can be written as

$$
\bar{G}(x ; \xi)=\frac{\left[\frac{1-u^{1 / b}}{\delta^{2}}\right]^{1 / 2}}{1+\bar{\delta}\left[\frac{1-u^{1 / b}}{\delta^{2}}\right]^{1 / 2}}
$$

which further simplifies to

$$
G(x ; \xi)=1-\left[\frac{\left[\frac{1-u^{1 / b}}{\delta^{2}}\right]^{1 / 2}}{1+\bar{\delta}\left[\frac{1-u^{1 / b}}{\delta^{2}}\right]^{1 / 2}}\right] .
$$

We can therefore determine the quantiles of the TL-MO-G family of distributions by solving the equation

$$
x(u)=G^{-1}\left[1-\left[\frac{\left[\frac{1-u^{1 / b}}{\delta^{2}}\right]^{1 / 2}}{1+\bar{\delta}\left[\frac{1-u^{1 / b}}{\delta^{2}}\right]^{1 / 2}}\right]\right],
$$

using iterative methods by making use of Matlab or R software. We present quantiles for the TL-MO-LLo distribution for some selected values of parameters. The results are shown in Table 2.

Table 2. Table of Quantiles for the TL-MO-LLo Distribution

\begin{tabular}{cccccc}
\hline $\mathrm{u}$ & $(1.5,1.5,1.5)$ & $(0.5,1,1.5)$ & $(1.1,0.5,0.5)$ & $(0.5,1.5,0.5)$ & $(0.9,1,0.9)$ \\
\hline 0.1 & 0.3345 & 0.0294 & 0.0011 & 0.0001 & 0.0288 \\
0.2 & 0.4959 & 0.0752 & 0.0049 & 0.0009 & 0.0739 \\
0.3 & 0.6460 & 0.1326 & 0.0128 & 0.0053 & 0.1345 \\
0.4 & 0.8023 & 0.2024 & 0.0273 & 0.0187 & 0.2155 \\
0.5 & 0.9771 & 0.2882 & 0.0535 & 0.0538 & 0.3259 \\
0.6 & 1.1863 & 0.3968 & 0.1026 & 0.1406 & 0.4830 \\
0.7 & 1.4581 & 0.5431 & 0.2026 & 0.3605 & 0.7245 \\
0.8 & 1.8590 & 0.7631 & 0.4447 & 1.0000 & 1.1499 \\
0.9 & 2.6277 & 1.1876 & 1.3327 & 3.7684 & 2.1705 \\
\hline
\end{tabular}




\section{Maximum Likelihood Estimation}

If $X_{i} \sim T L-M O-G(b, \delta ; \xi)$ with the parameter vector $\Delta=(b, \delta ; \xi)^{T}$. The total $\log$-likelihood $\ell=\ell(\Delta)$ from a random sample of size $n$ is given by

$$
\begin{aligned}
\ell & =n \log (2 b)+2 n \log (\delta)+\sum_{i=1}^{n} \log \left[g\left(x_{i} ; \xi\right)\right]+\sum_{i=1}^{n} \log \left[\bar{G}\left(x_{i} ; \xi\right)\right] \\
& -3 \sum_{i=1}^{n} \log \left[1-\bar{\delta} \bar{G}\left(x_{i} ; \xi\right)\right]+(b-1) \sum_{i=1}^{n} \log \left[1-\frac{\delta^{2} \bar{G}^{2}\left(x_{i} ; \xi\right)}{\left(1-\bar{\delta} \bar{G}\left(x_{i} ; \xi\right)\right)^{2}}\right]
\end{aligned}
$$

The score vector $U=\left(\frac{\partial \ell}{\partial b}, \frac{\partial \ell}{\partial \delta}, \frac{\partial \ell}{\partial \xi_{k}}\right)$ has elements given by:

$$
\frac{\partial \ell}{\partial b}=\frac{n}{b}+\sum_{i=1}^{n} \log \left[1-\frac{\delta^{2} \bar{G}^{2}\left(x_{i} ; \xi\right)}{\left(1-\bar{\delta} \bar{G}\left(x_{i} ; \xi\right)\right)^{2}}\right]
$$

$$
\begin{aligned}
\frac{\partial \ell}{\partial \delta} & =\frac{2 n}{\delta}-3 \sum_{i=1}^{n} \frac{\bar{G}\left(x_{i} ; \xi\right)}{\left[1-\bar{\delta} \bar{G}\left(x_{i} ; \xi\right)\right]} \\
& -(b-1) \sum_{i=1}^{n} \frac{2 \delta \bar{G}^{2}\left(x_{i} ; \xi\right) G\left(x_{i} ; \xi\right)}{\left(\bar{G}^{2}\left(x_{i} ; \xi\right)-2 \delta \bar{G}^{2}\left(x_{i} ; \xi\right)+2 \delta \bar{G}\left(x_{i} ; \xi\right)-2 \bar{G}\left(x_{i} ; \xi\right)+1\right)\left(1-\bar{\delta} \bar{G}\left(x_{i} ; \xi\right)\right)}
\end{aligned}
$$

and

$$
\begin{aligned}
\frac{\partial \ell}{\partial \xi_{k}} & =\sum_{i=1}^{n} \frac{1}{g\left(x_{i} ; \xi\right)} \frac{\partial g\left(x_{i} ; \xi\right)}{\partial \xi_{k}}+\sum_{n=1}^{n} \frac{1}{\bar{G}\left(x_{i} ; \xi\right)} \frac{\partial \bar{G}\left(x_{i} ; \xi\right)}{\partial \xi_{k}}-3 \sum_{i=1}^{n} \frac{1}{\left[1-\bar{\delta} \bar{G}\left(x_{i} ; \xi\right)\right]} \frac{\partial\left[1-\bar{\delta} \bar{G}\left(x_{i} ; \xi\right)\right]}{\partial \xi_{k}} \\
& +(b-1) \sum_{i=1}^{n} \frac{1}{\left[1-\frac{\delta^{2} \bar{G}^{2}\left(x_{i} ; \xi\right)}{\left(1-\bar{\delta} \bar{G}\left(x_{i} ; \xi\right)\right)^{2}}\right]} \frac{\partial\left[1-\frac{\delta^{2} \bar{G}^{2}\left(x_{i} ; \xi\right)}{\left(1-\bar{\delta} \bar{G}\left(x_{i} ; \xi\right)\right)^{2}}\right]}{\partial \xi_{k}},
\end{aligned}
$$

respectively. These partial derivatives are not in closed form and can be solved using R, MATLAB and SAS software by use of iterative methods.

\section{Simulation Study}

We conducted a simulation study to evaluate consistency of the maximum likelihood estimators. We simulated for $\mathrm{N}=1000$ times with sample size $n=60,120,240,480,960$ and 1920. Simulation results are shown in Table 3. From the Monte Carlo simulation results, we conclude that our model produces consistent results when estimating parameters for the model because as the sample size increases the mean result approaches the true parameters values and also the root mean square error (RMSE) and average bias dies towards zero for all parrameters values. 
Table 3. Monte Carlo Simulation Results for TL-MO-LLo Distribution: Mean, RMSE and Average Bias

\begin{tabular}{|c|c|c|c|c|c|c|c|}
\hline & I & \multicolumn{3}{|c|}{$b=1.1, \delta=1.1, c=1.1$} & \multicolumn{2}{|r|}{$b=1.1, \delta=0.5, c=1.1$} & \multirow[b]{2}{*}{ Bias } \\
\hline Parameter & $n$ & Mean & RMSE & Bias & Mean & RMSE & \\
\hline \multirow{6}{*}{$b$} & 60 & 1.671536 & 2.716772 & 0.571536 & 1.624344 & 2.215333 & 0.524344 \\
\hline & 120 & 1.269711 & 0.715419 & 0.169712 & 1.275546 & 0.729886 & 0.175546 \\
\hline & 240 & 1.162377 & 0.346449 & 0.062377 & 1.164539 & 0.341169 & 0.064539 \\
\hline & 480 & 1.141683 & 0.237301 & 0.041683 & 1.142029 & 0.238003 & 0.042029 \\
\hline & 960 & 1.112831 & 0.155251 & 0.012831 & 1.113683 & 0.155635 & 0.013683 \\
\hline & 1920 & 1.109277 & 0.105180 & 0.009277 & 1.111168 & 0.105385 & 0.011168 \\
\hline \multirow{6}{*}{$\delta$} & 60 & 1.541142 & 6.027015 & 0.441142 & 0.505367 & 0.264703 & 0.005367 \\
\hline & 120 & 1.167736 & 0.516749 & 0.067736 & 0.496512 & 0.146580 & -0.003488 \\
\hline & 240 & 1.146236 & 0.337147 & 0.046236 & 0.503414 & 0.101056 & 0.003414 \\
\hline & 480 & 1.106337 & 0.211200 & 0.006337 & 0.496897 & 0.068617 & -0.003103 \\
\hline & 960 & 1.109369 & 0.154057 & 0.009369 & 0.500067 & 0.049214 & 0.000067 \\
\hline & 1920 & 1.100255 & 0.103020 & 0.000255 & 0.498229 & 0.033279 & -0.001771 \\
\hline \multirow{6}{*}{$c$} & 60 & 1.166915 & 0.386696 & 0.066915 & 1.154401 & 0.361634 & 0.054401 \\
\hline & 120 & 1.135272 & 0.214548 & 0.035272 & 1.132911 & 0.210466 & 0.032911 \\
\hline & 240 & 1.117426 & 0.151956 & 0.017426 & 1.115446 & 0.149430 & 0.015446 \\
\hline & 480 & 1.104552 & 0.100178 & 0.004552 & 1.104568 & 0.100504 & 0.004568 \\
\hline & 960 & 1.104761 & 0.071815 & 0.004761 & 1.104517 & 0.071968 & 0.004517 \\
\hline & 1920 & 1.100690 & 0.049403 & 0.000690 & 1.099987 & 0.049394 & -0.000013 \\
\hline & III & \multicolumn{3}{|c|}{$b=2.0, \delta=0.7, c=0.7$} & IV & $b=1.7, \delta=1.7, c=1.7$ & \\
\hline \multirow{6}{*}{$b$} & 60 & 9.199453 & 60.488787 & 7.199453 & 6.028331 & 35.941240 & 4.328331 \\
\hline & 120 & 2.859115 & 4.110470 & 0.859115 & 2.215888 & 2.213599 & 0.515888 \\
\hline & 240 & 2.228598 & 0.937163 & 0.228598 & 1.855684 & 0.698894 & 0.155684 \\
\hline & 480 & 2.137067 & 0.615844 & 0.137067 & 1.797555 & 0.467849 & 0.097555 \\
\hline & 960 & 2.047915 & 0.366824 & 0.047915 & 1.732823 & 0.287797 & 0.032823 \\
\hline & 1920 & 2.028437 & 0.244751 & 0.028437 & 1.720528 & 0.193651 & 0.020528 \\
\hline \multirow{6}{*}{$\delta$} & 60 & 0.767782 & 0.770846 & 0.067782 & 3.524084 & 26.950170 & 1.824084 \\
\hline & 120 & 0.718927 & 0.308559 & 0.018927 & 1.904017 & 1.132744 & 0.204017 \\
\hline & 240 & 0.717434 & 0.212059 & 0.017434 & 1.823910 & 0.731741 & 0.123911 \\
\hline & 480 & 0.699030 & 0.140029 & -0.000970 & 1.728074 & 0.444520 & 0.028074 \\
\hline & 960 & 0.703179 & 0.100900 & 0.003179 & 1.726121 & 0.320305 & 0.026121 \\
\hline & 1920 & 0.699275 & 0.068543 & -0.000725 & 1.705366 & 0.215132 & 0.005366 \\
\hline \multirow{7}{*}{$c$} & 60 & 0.722590 & 0.200347 & 0.022590 & 1.776246 & 0.516945 & 0.076246 \\
\hline & 120 & 0.713894 & 0.124577 & 0.013894 & 1.743015 & 0.313702 & 0.043015 \\
\hline & 240 & 0.706694 & 0.090913 & 0.006694 & 1.721157 & 0.225319 & 0.021157 \\
\hline & 480 & 0.701539 & 0.061989 & 0.001539 & 1.704685 & 0.151382 & 0.004685 \\
\hline & 960 & 0.701972 & 0.043573 & 0.001972 & 1.705606 & 0.106903 & 0.005606 \\
\hline & 1920 & 0.700272 & 0.030339 & 0.000272 & 1.700826 & 0.074252 & 0.000826 \\
\hline & $\mathrm{V}$ & \multicolumn{3}{|c|}{$b=1.2, \delta=1.7, c=1.7$} & VI & $b=1.7, \delta=1.7, c=1.2$ & \\
\hline \multirow{6}{*}{$b$} & 60 & 1.980490 & 4.372702 & 0.780490 & 5.297957 & 31.003670 & 3.597957 \\
\hline & 120 & 1.408795 & 0.877816 & 0.208795 & 2.159727 & 1.941070 & 0.459727 \\
\hline & 240 & 1.272933 & 0.392300 & 0.072933 & 1.860091 & 0.705019 & 0.160091 \\
\hline & 480 & 1.249058 & 0.270021 & 0.049058 & 1.796749 & 0.467161 & 0.096749 \\
\hline & 960 & 1.215140 & 0.174880 & 0.015140 & 1.731816 & 0.286004 & 0.031816 \\
\hline & 1920 & 1.210590 & 0.118698 & 0.010590 & 1.720531 & 0.193642 & 0.020531 \\
\hline \multirow{6}{*}{$\delta$} & 60 & 3.757668 & 35.337334 & 2.057668 & 3.059995 & 20.948873 & 1.359995 \\
\hline & 120 & 1.903173 & 1.077633 & 0.203173 & 1.913688 & 1.141291 & 0.213688 \\
\hline & 240 & 1.815955 & 0.662518 & 0.115955 & 1.819768 & 0.729411 & 0.119768 \\
\hline & 480 & 1.725430 & 0.398814 & 0.025430 & 1.728600 & 0.444425 & 0.028600 \\
\hline & 960 & 1.724927 & 0.290622 & 0.024927 & 1.726743 & 0.319865 & 0.026743 \\
\hline & 1920 & 1.704527 & 0.193927 & 0.004527 & 1.705360 & 0.215126 & 0.005360 \\
\hline \multirow{6}{*}{$c$} & 60 & 1.804810 & 0.585982 & 0.104810 & 1.256652 & 0.361618 & 0.056652 \\
\hline & 120 & 1.754136 & 0.329008 & 0.054136 & 1.231874 & 0.220701 & 0.031874 \\
\hline & 240 & 1.726360 & 0.231808 & 0.026360 & 1.214045 & 0.158588 & 0.014045 \\
\hline & 480 & 1.706420 & 0.153738 & 0.006420 & 1.203355 & 0.106898 & 0.003355 \\
\hline & 960 & 1.706993 & 0.109837 & 0.006993 & 1.204081 & 0.075396 & 0.004081 \\
\hline & 1920 & 1.701127 & 0.075905 & 0.001127 & 1.200581 & 0.052413 & 0.000581 \\
\hline
\end{tabular}




\section{Applications}

We applied the TL-MO-LLo model to three real data examoles to demonstrate usefulness of the new distribution compared to its sub-models models and other known non-nested distributions. The best fitting model was assessed using the goodness-of-fit statistics, namely, -2loglikelihood (-2 log L), Akaike Information Criterion (AIC), Consistent Akaike Information Criterion (AICC), Bayesian Information Criterion (BIC), Cramer von Mises $\left(W^{*}\right)$ and Andersen-Darling $\left(A^{*}\right)$ as described by (Chen and Balakrishnan, 1995). The best model has smaller values of these statistics. We used R software to estimate the model parameters via the nlm function. Model parameter estimates (standard errors in parenthesis) and the goodness-of-fit-statistics for the three data sets are shown in Tables 4, 5 and 6. We also present plots of the fitted densities, the histogram of the data and probability plots (Chambers, Cleveland, Kleiner and Tukey, 1983) to show how well our model fits the observed data sets. The plots are shown in Figures 5, 6 and 7.

We compared the TL-MO-LLo distribution with other competing three parameter non-nested models, namely MarshallOlkin extended inverse Weibull (IWMO) by (Pakungwati, Widyaningsih and Lestari, 2018) and the exponentiated-Fréchet (EFr) distribution by (Nadarajah and Kotz, 2003).

Furthermore, the TL-MO-LLo model is compared to the other two non-nested studied by (Barreto-Souza, Lemonte and Cordeiro, 2013), namely, Marshall-Olkin extended Fréchet (MOEFr) and Marshall-Olkin extended generalized exponential (MOEGE) distributions. Furthermore, we compared the TL-MO-LLo distribution to other three non-nested models by (Hassan, Elgarhy and Zubair, 2019) : type II generalized Topp-Leone-uniform (TIGTLU), type II generalized Topp-Leone-exponential (TIGTLE) and type II generalized Topp-Leone-Rayleigh (TIGTLR). The pdfs of the non-nested models are given by:

$$
f_{\text {IШмо }}(x ; \alpha, \theta \lambda)=\frac{\alpha \lambda \theta^{-\lambda} x^{-\lambda-1} e^{-(\theta x)^{-\lambda}}}{\left[\alpha-(\alpha-1) e^{-(\theta x)^{-\lambda}}\right]^{2}},
$$

for $\alpha, \theta, \lambda>0$,

$$
f_{E F r}(x ; \alpha, \lambda, \delta)=\alpha \lambda \delta^{\lambda}\left[1-e^{-(\delta / x)^{\lambda}}\right]^{\alpha-1} x^{-(1+\lambda)} e^{-(\lambda+1)(\delta / x)^{\lambda}},
$$

for $\alpha, \lambda, \delta>0$,

$$
f_{\text {MOEFr }}(x ; \alpha, \lambda, \delta)=\frac{\alpha \lambda \delta^{\lambda} x^{-(\lambda+1)} e^{-(\delta / x)^{\lambda}}}{\left[1-\bar{\alpha}\left(1-e^{-(\delta / x)^{\lambda}}\right)\right]^{2}},
$$

for $\alpha, \lambda, \delta>0$,

$$
f_{M O E G E}(x ; \alpha, \gamma, \lambda)=\frac{\alpha \gamma \lambda e^{-\lambda x}\left(1-e^{-\lambda x}\right)^{\gamma-1}}{\left(1-\bar{\alpha}\left[1-e^{-\lambda x}\right]^{\gamma}\right)^{2}},
$$

for $\alpha, \gamma, \lambda>0$,

$$
f_{\text {TIIGTLU }}(x ; \alpha, \beta, \theta)=\frac{2 \alpha \beta}{\theta}\left(\frac{x}{\theta}\right)^{2 \beta-1}\left(1-\frac{x}{\theta}\right)^{\alpha-1},
$$

for $\alpha, \beta, \theta>0$,

$$
f_{\text {TIGTLE }}(x ; \alpha, \beta, \theta)=2 \alpha \beta \theta e^{\theta x}\left[1-e^{-\theta x}\right]^{2 \beta-1}\left(1-\left(1-e^{-\theta x}\right)^{2 \beta}\right)^{\alpha-1},
$$

for $\alpha, \beta, \theta>0$ and

$$
f_{\text {TIGTLR }}(x ; \alpha, \beta, \theta)=4 \alpha \beta \theta x e^{\theta x^{2}}\left[1-e^{-\theta x^{2}}\right]^{2 \beta-1}\left(1-\left(1-e^{-\theta x^{2}}\right)^{2 \beta}\right)^{\alpha-1},
$$

for $\alpha, \beta, \theta>0$.

\subsection{Kevlar 49/Epoxy Strands Failure at 90\% Data}

The first data set consists of 101 observations representing failure times (in hours) of kevlar 49/epoxy strands subjected to constant sustained pressure at the 90\% stress level (see Andrews and Herzberg, 2012 or Barlow, Toland and Freeman, 1984 for details). $0.01,0.01,0.02,0.02,0.02,0.03,0.03,0.04,0.05,0.06,0.07,0.07,0.08,0.09,0.09,0.10,0.10,0.11$, $0.11,0.12,0.13,0.18,0.19,0.20,0.23,0.24,0.24,0.29,0.34,0.35,0.36,0.38,0.40,0.42,0.43,0.52,0.54,0.56,0.60$, $0.60,0.63,0.65,0.67,0.68,0.72,0.72,0.72,0.73,0.79,0.79,0.80,0.80,0.83,0.85,0.90,0.92,0.95,0.99,1.00,1.01$, $1.02,1.03,1.05,1.10,1.10,1.11,1.15,1.18,1.20,1.29,1.31,1.33,1.34,1.40,1.43,1.45,1.50,1.51,1.52,1.53,1.54$, $1.54,1.55,1.58,1.60,1.63,1.64,1.80,1.80,1.81,2.02,2.05,2.14,2.17,2.33,3.03,3.03,3.34,4.20,4.69,7.89$.

The estimated variance-covariance matrix for TL-MO-LLo model on kevlar data set is given by

$$
\left[\begin{array}{ccc}
0.00798 & -0.37564 & -0.03881 \\
-0.37564 & 21.06806 & 1.96500 \\
-0.03881 & 1.96500 & 0.23179
\end{array}\right]
$$


and the $95 \%$ confidence intervals for the model parameters are given by $b \in[0.3169 \pm 0.1751], \delta \in[7.5481 \pm 8.9964]$ and $c \in[2.3460 \pm 0.9436]$.

Table 4. Parameter estimates and goodness of fit statistics for various models fitted for kevlar data set

\begin{tabular}{|c|c|c|c|c|c|c|c|c|c|c|c|}
\hline \multirow[b]{2}{*}{ Model } & \multicolumn{4}{|c|}{ Estimates } & \multicolumn{4}{|c|}{ Statistics } & \multirow[b]{2}{*}{$A^{*}$} & \multirow[b]{2}{*}{$\mathrm{K}-\mathrm{S}$} & \multirow[b]{2}{*}{ p-value } \\
\hline & $b$ & $\delta$ & $c$ & $-2 \log L$ & $A I C$ & $A I C C$ & $B I C$ & $W^{*}$ & & & \\
\hline TL-MO-LLo & $\begin{array}{c}0.3169 \\
(0.0893)\end{array}$ & $\begin{array}{c}7.5481 \\
(4.5899)\end{array}$ & $\begin{array}{c}2.3460 \\
(0.4815)\end{array}$ & 202.1 & 208.1 & 208.3 & 215.9 & 0.0972 & 0.6206 & 0.0645 & 0.7945 \\
\hline TL-MO-LLo $(1, \delta, c)$ & $\begin{array}{l}1 \\
-\end{array}$ & $\begin{array}{c}1.4542 \\
(0.2006)\end{array}$ & $\begin{array}{c}1.1351 \\
(0.0949)\end{array}$ & 214.0 & 218.0 & 218.1 & 223.2 & 0.3836 & 2.0777 & 0.1039 & 0.2261 \\
\hline $\operatorname{TL}-\mathrm{MO}-\mathrm{LLo}(b, 1, c)$ & $\begin{array}{c}1.2491 \\
(0.1773)\end{array}$ & $\begin{array}{l}1 \\
-\end{array}$ & $\begin{array}{c}1.0120 \\
(0.1165)\end{array}$ & 219.1 & 223.1 & 223.2 & 228.3 & 0.4733 & 2.5597 & 0.1604 & 0.0111 \\
\hline TL-MO-LLo $(b, \delta, 1)$ & $\begin{array}{c}1.0317 \\
(0.1580)\end{array}$ & $\begin{array}{c}1.4163 \\
(0.2929)\end{array}$ & $\begin{array}{l}1 \\
-\end{array}$ & 216.2 & 220.2 & 220.3 & 225.4 & 0.4159 & 2.2457 & 0.1196 & 0.1114 \\
\hline TL-MO-LLo $(b, 1,1)$ & $\begin{array}{c}1.2624 \\
(0.1256)\end{array}$ & $\begin{array}{l}1 \\
-\end{array}$ & $\begin{array}{l}1 \\
-\end{array}$ & 219.1 & 221.1 & 221.2 & 223.7 & 0.4766 & 2.5766 & 0.1589 & 0.0122 \\
\hline TL-MO-LLo $(1, \delta, 1)$ & $\begin{array}{l}1 \\
-\end{array}$ & $\begin{array}{c}1.4623 \\
(0.1982)\end{array}$ & $\begin{array}{l}1 \\
-\end{array}$ & 216.1 & 218.2 & 218.2 & 220.8 & 0.4101 & 2.2138 & 0.1209 & 0.1040 \\
\hline TL-MO-LLo $(1,1, c)$ & $\begin{array}{l}1 \\
-\end{array}$ & $\begin{array}{l}1 \\
- \\
\end{array}$ & $\begin{array}{c}1.1480 \\
(0.0935) \\
\end{array}$ & 221.5 & 223.5 & 223.5 & 226.1 & 0.4269 & 2.3163 & 0.2125 & 0.0002 \\
\hline MOEGE & $\begin{array}{c}\alpha \\
0.5942 \\
(0.3306)\end{array}$ & $\begin{array}{c}\gamma \\
0.7307 \\
(0.1849)\end{array}$ & $\begin{array}{c}\lambda \\
1.0456 \\
(0.2071)\end{array}$ & 204.8 & 210.8 & 211.0 & 218.6 & 0.0946 & 0.9496 & 0.4269 & $<2.200 \times 10^{-16}$ \\
\hline $\mathrm{EFr}$ & $\begin{array}{c}\alpha \\
0.5753 \\
(0.2606)\end{array}$ & $\begin{array}{c}\delta \\
0.0534 \\
(0.0343)\end{array}$ & $\begin{array}{c}\lambda \\
0.6869 \\
(0.1889)\end{array}$ & 353.3 & 359.3 & 359.6 & 367.2 & 1.1458 & 6.1972 & 0.2780 & $3.3100 \times 10^{-7}$ \\
\hline MOEFr & $\begin{array}{c}312.85 \\
\left(4.4822 \times 10^{-6}\right)\end{array}$ & $\begin{array}{c}0.0066 \\
\left(2.6808 \times 10^{-3}\right)\end{array}$ & $\begin{array}{c}1.2631 \\
(0.1033)\end{array}$ & 225.2 & 231.2 & 231.5 & 239.1 & 33.5614 & 199.4421 & 0.9960 & $<2.200 \times 10^{-16}$ \\
\hline IWMO & $\begin{array}{c}\alpha \\
312.8554 \\
(9.7035) \\
\end{array}$ & $\begin{array}{c}\lambda \\
1.2631 \\
(0.1041) \\
\end{array}$ & $\begin{array}{c}\theta \\
152.0403 \\
(54.9189) \\
\end{array}$ & 225.2 & 231.2 & 231.5 & 239.1 & 0.5761 & 3.1182 & 0.1121 & 0.1579 \\
\hline TIIGTLU & $\begin{array}{c}\alpha \\
185.9870 \\
(53.9118)\end{array}$ & $\begin{array}{c}\beta \\
0.4612 \\
(0.0365)\end{array}$ & $\begin{array}{c}\theta \\
286.8642 \\
(32.9891)\end{array}$ & 205.9 & 211.9 & 212.2 & 219.8 & 33.5301 & 201.2281 & 0.9989 & $<2.200 \times 10^{-16}$ \\
\hline TIIGTLE & $\begin{array}{c}0.3247 \\
(0.6552)\end{array}$ & $\begin{array}{c}0.3988 \\
(0.1097)\end{array}$ & $\begin{array}{c}2.8242 \\
(5.8353)\end{array}$ & 205.3 & 211.3 & 211.6 & 219.2 & 0.1599 & 0.9329 & 0.0842 & 0.4696 \\
\hline TIIGTLR & $\begin{array}{c}66.8640 \\
\left(6.5275 \times 10^{-7}\right)\end{array}$ & $\begin{array}{c}0.2291 \\
(0.0178)\end{array}$ & $\begin{array}{c}0.0001 \\
\left(7.0156 \times 10^{-5}\right)\end{array}$ & 205.9 & 211.9 & 212.2 & 219.8 & 0.1957 & 1.0982 & 0.0848 & 0.4616 \\
\hline
\end{tabular}
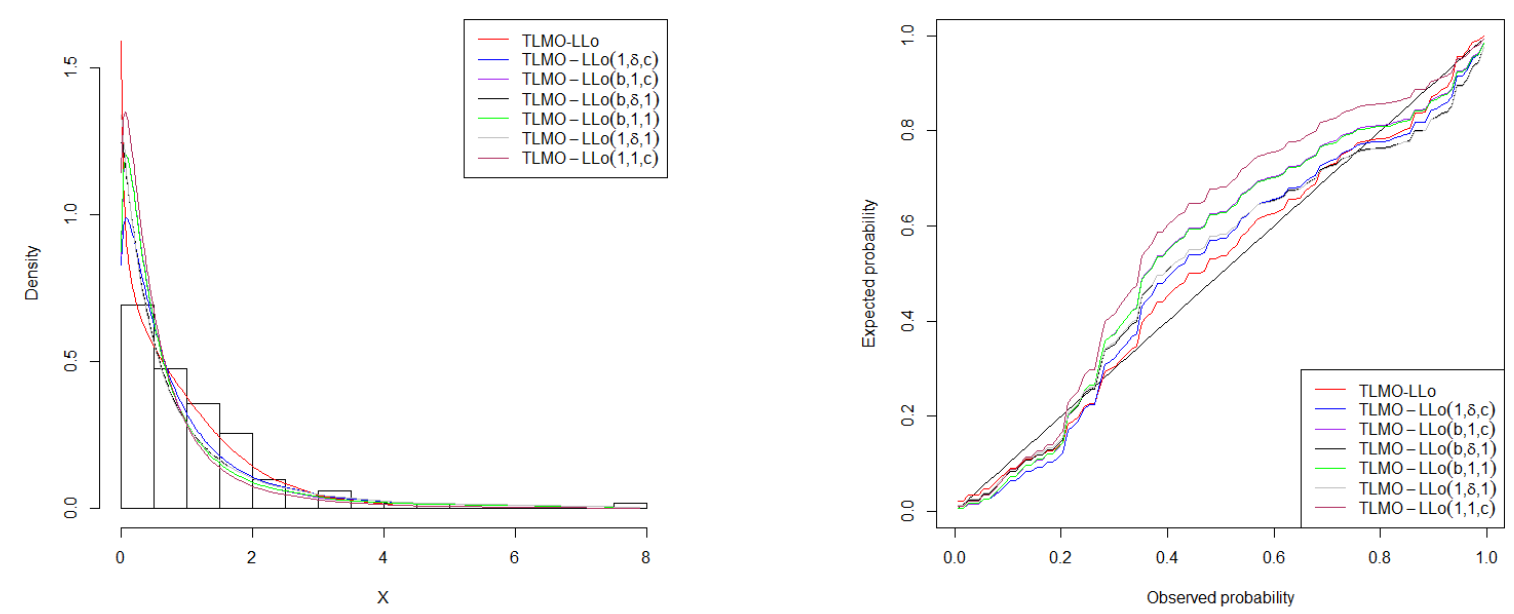

Figure 5. Fitted pdfs and probability plots for kevlar data set

From the results shown in Table 4, we can conclude that the TL-MO-LLo distribution fit the kevlar data set better than the non-nested models considered. Furthermore, from the fitted densities plots (Figure 5), we can conclude that the proposed model fits well on data that is heavy-tailed compared to the sub-models.

\subsection{Strengths of $1.5 \mathrm{~cm}$ Glass Fibres Data}

The second data set was analyzed by by (Bourguignon, Silva and Cordeiro, 2014) and (Smith and Naylor, 1987) and represents the strengths of $1.5 \mathrm{~cm}$ glass fibres. The observations are as follows: $0.55,0.93,1.25,1.36,1.49,1.52,1.58$, 
$1.61,1.64,1.68,1.73,1.81,2.00,0.74,1.04,1.27,1.39,1.49,1.53,1.59,1.61,1.66,1.68,1.76,1.82,2.01,0.77,1.11$, $1.28,1.42,1.50,1.54,1.60,1.62,1.66,1.69,1.76,1.84,2.24,0.81,1.13,1.29,1.48,1.50,1.55,1.61,1.62,1.66,1.70$, $1.77,1.84,0.84,1.24,1.30,1.48,1.51,1.55,1.61,1.63,1.67,1.70,1.78,1.89$.

The estimated variance-covariance matrix for TL-MO-LLo model on $1.5 \mathrm{~cm}$ glass fibres data set is given by

$$
\left[\begin{array}{ccc}
1.77699 \times 10^{-3} & -1.14663 \times 10^{-6} & 7.40487 \times 10^{-3} \\
-1.14663 \times 10^{-6} & 6.87674 \times 10^{-9} & -4.41496 \times 10^{-5} \\
7.40487 \times 10^{-3} & -4.41496 \times 10^{-5} & 2.83448 \times 10^{-1}
\end{array}\right]
$$

and the $95 \%$ confidence intervals for the model parameters are given by $b \in[0.3159 \pm 0.0826], \delta \in[9521.6 \pm 0.00016]$ and $c \in[14.7220 \pm 1.0435]$.

Table 5. Parameter estimates and goodness of fit statistics for various models fitted for $1.5 \mathrm{~cm}$ glass fibres data set

\begin{tabular}{|c|c|c|c|c|c|c|c|c|c|c|c|}
\hline \multirow[b]{2}{*}{ Model } & \multicolumn{4}{|c|}{ Estimates } & \multicolumn{4}{|c|}{ Statistics } & \multirow[b]{2}{*}{$A^{*}$} & \multirow[b]{2}{*}{ K-S } & \multirow[b]{2}{*}{ p-value } \\
\hline & $b$ & $\delta$ & $c$ & $-2 \log L$ & $A I C$ & $A I C C$ & $B I C$ & $W^{*}$ & & & \\
\hline TL-MO-LLo & $\begin{array}{c}0.3159 \\
(0.0422)\end{array}$ & $\begin{array}{c}9.5216 \times 10^{3} \\
\left(8.2926 \times 10^{-5}\right)\end{array}$ & $\begin{array}{l}14.7220 \\
(0.5324)\end{array}$ & 25.5 & 31.5 & 31.9 & 37.9 & 0.1372 & 0.7608 & 0.1343 & 0.2056 \\
\hline TL-MO-LLo $(1, \delta, c)$ & $\begin{array}{l}1 \\
-\end{array}$ & $\begin{array}{c}48.9084 \\
(20.1471)\end{array}$ & $\begin{array}{c}7.1352 \\
(0.7745)\end{array}$ & 36.7 & 40.7 & 40.9 & 45.0 & 0.3582 & 1.9723 & 0.1535 & 0.1029 \\
\hline TL-MO-LLo $(b, 1, c)$ & $\begin{array}{c}8.2359 \\
(1.0717)\end{array}$ & $\begin{array}{l}1 \\
-\end{array}$ & $\begin{array}{c}2.5269 \\
(0.1972)\end{array}$ & 71.1 & 75.1 & 75.3 & 79.4 & 0.9220 & 5.0111 & 0.2484 & 0.0008 \\
\hline $\operatorname{TL}-\operatorname{MO}-\operatorname{LLo}(b, \delta, 1)$ & $\begin{array}{c}3.4862 \times 10^{5} \\
\left(4.6404 \times 10^{-13}\right)\end{array}$ & $\begin{array}{c}2.2635 \times 10^{-3} \\
\left(1.4311 \times 10^{-4}\right)\end{array}$ & $\begin{array}{l}1 \\
-\end{array}$ & 109.4 & 113.4 & 113.6 & 117.7 & 1.0444 & 5.5991 & 0.3457 & $5.57710 \times 10^{-7}$ \\
\hline $\operatorname{TL}-\mathrm{MO}-\operatorname{LLo}(b, 1,1)$ & $\begin{array}{c}5.3080 \\
(0.6688)\end{array}$ & $\begin{array}{l}1 \\
-\end{array}$ & $\begin{array}{l}1 \\
-\end{array}$ & 148.5 & 150.5 & 150.6 & 152.7 & 0.7534 & 4.1124 & 0.4466 & $2.4340 \times 10^{-11}$ \\
\hline TL-MO-LLo $(1, \delta, 1)$ & $\begin{array}{l}1 \\
-\end{array}$ & $\begin{array}{c}2.9636 \\
(0.4601)\end{array}$ & $\begin{array}{l}1 \\
-\end{array}$ & 203.9 & 205.9 & 206.0 & 208.1 & 0.6503 & 3.5619 & 0.3601 & $1.6050 \times 10^{-7}$ \\
\hline TL-MO-LLo $(1,1, c)$ & $\begin{array}{l}1 \\
-\end{array}$ & $\begin{array}{l}1 \\
-\end{array}$ & $\begin{array}{c}2.0520 \\
(0.2108)\end{array}$ & 221.5 & 223.5 & 223.6 & 225.7 & 0.7177 & 3.9305 & 0.7039 & $<2.200 \times 10^{-16}$ \\
\hline MOEGE & $\begin{array}{c}\alpha \\
1.3296 \times 10^{-3} \\
(0.3306)\end{array}$ & $\begin{array}{c}\gamma \\
10.7200 \\
(0.1849)\end{array}$ & $\begin{array}{c}\lambda \\
5.8366 \\
(0.2071)\end{array}$ & 31.9 & 37.9 & 38.4 & 44.4 & 0.4410 & 2.4309 & 0.9995 & $<2.200 \times 10^{-16}$ \\
\hline $\mathrm{EFr}$ & $\begin{array}{c}\alpha \\
0.04621 \\
(0.0153)\end{array}$ & $\begin{array}{c}\delta \\
0.4993 \\
(0.0147)\end{array}$ & $\begin{array}{c}\lambda \\
20.1145 \\
(6.1482)\end{array}$ & 189.1 & 195.1 & 195.5 & 201.6 & 1.1986 & 6.3098 & 0.4279 & $1.9210 \times 10^{-10}$ \\
\hline MOEFr & $\begin{array}{c}54074 \\
\left(3.8277 \times 10^{-8}\right)\end{array}$ & $\begin{array}{c}0.3858 \\
\left(6.0532 \times 10^{-2}\right)\end{array}$ & $\begin{array}{c}7.9253 \\
(0.8731)\end{array}$ & 45.6 & 51.6 & 51.9 & 58.0 & 19.2509 & 122.7666 & 0.9997 & $<2.200 \times 10^{-16}$ \\
\hline IWMO & $\begin{array}{c}\alpha \\
52636 \\
(9.7035)\end{array}$ & $\begin{array}{c}\lambda \\
7.9256 \\
(0.1041)\end{array}$ & $\begin{array}{c}\theta \\
2.5828 \\
(54.9189)\end{array}$ & 45.6 & 51.6 & 51.9 & 58.0 & 0.4974 & 2.7509 & 0.1536 & 0.1020 \\
\hline TIIGTLU & $\begin{array}{c}\alpha \\
48.4266 \\
(336.0839)\end{array}$ & $\begin{array}{c}\beta \\
2.8465 \\
(0.4190)\end{array}$ & $\begin{array}{c}\theta \\
3.2268 \\
(3.6401)\end{array}$ & 30.4 & 36.4 & 36.8 & 42.8 & 19.4750 & 124.3383 & 0.9985 & $<2.200 \times 10^{-16}$ \\
\hline TIIGTLE & $\begin{array}{c}530890 \\
\left(1.5076 \times 10^{-8}\right)\end{array}$ & $\begin{array}{c}3.0526 \\
(0.3499)\end{array}$ & $\begin{array}{c}0.0754 \\
(0.0195)\end{array}$ & 30.8 & 36.8 & 37.2 & 43.2 & 0.2464 & 1.3526 & 0.1549 & 0.0975 \\
\hline TIIGTLR & $\begin{array}{c}52242 \\
\left(1.5128 \times 10^{-8}\right)\end{array}$ & $\begin{array}{c}1.4595 \\
(0.1519)\end{array}$ & $\begin{array}{c}0.0092 \\
\left(3.5319 \times 10^{-5}\right)\end{array}$ & 30.5 & 36.5 & 36.9 & 42.9 & 0.2410 & 1.3238 & 0.1485 & 0.1241 \\
\hline
\end{tabular}

We can also conclude from the results shown in Table 5 that the TL-MO-LLo distribution fit the glass fibres data set better than the non-nested models considered. The TL-MO-LLo distribution has smaller values of the the goodness-of-fit statistics and a bigger p-value for the K-S statistic. Furthermore, from the fitted densities plots (Figure 6), we can notice the improvement achieved by using the TL-MO-LLo distribution in fitting the glass fibre data compared to the sub-models.

\subsection{Silicon Nitride Data}

The third data set represents fracture toughness of silicon nitride measured in MPa $m^{1 / 2}$. The data set was also analyzed by (Nadarajah and Kotz, 2007) and also by (Ali, Hasnain and Ahmad, 2015). The data are 5.50, 5.00, 4.90, 6.40, 5.10, 5.20, 5.20, 5.00, 4.70, 4.00, 4.50, 4.20, 4.10, 4.56, 5.01, 4.70, 3.13, 3.12, 2.68, 2.77, 2.70, 2.36, 4.38, 5.73, 4.35, 6.81, $1.91,2.66,2.61,1.68,2.04,2.08,2.13,3.80,3.73,3.71,3.28,3.90,4.00,3.80,4.10,3.90,4.05,4.00,3.95,4.00,4.50$, $4.50,4.20,4.55,4.65,4.10,4.25,4.30,4.50,4.70,5.15,4.30,4.50,4.90,5.00,5.35,5.15,5.25,5.80,5.85,5.90,5.75$, $6.25,6.05,5.90,3.60,4.10,4.50,5.30,4.85,5.30,5.45,5.10,5.30,5.20,5.30,5.25,4.75,4.50,4.20,4.00,4.15,4.25$, $4.30,3.75,3.95,3.51,4.13,5.40,5.00,2.10,4.60,3.20,2.50,4.10,3.50,3.20,3.30,4.60,4.30,4.30,4.50,5.50,4.60$, $4.90,4.30,3.00,3.40,3.70,4.40,4.90,4.90,5.00$. 

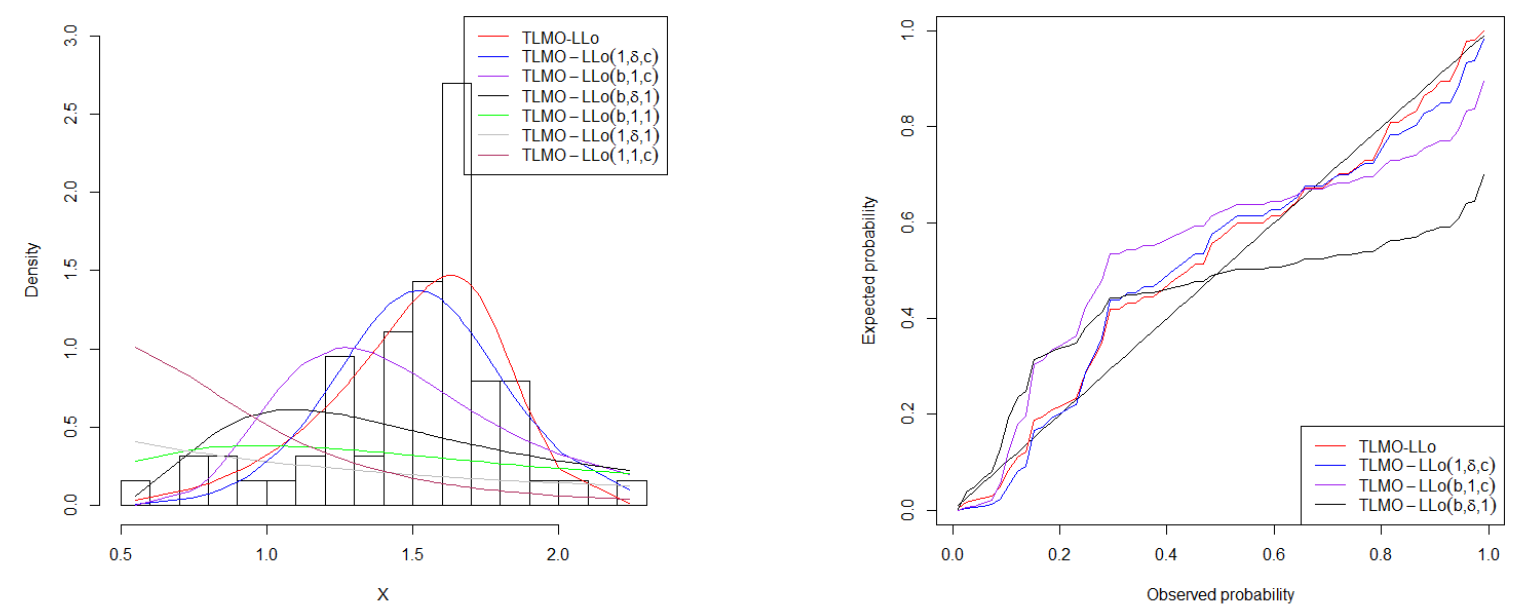

Figure 6. Fitted pdfs and probability plots for glass fibres data set

The estimated variance-covariance matrix for TL-MO-LLo model on silicon nitride data set is given by

$$
\left[\begin{array}{ccc}
1.85890 \times 10^{-3} & -2.98495 \times 10^{-11} & 3.06163 \times 10^{-3} \\
-2.98495 \times 10^{-11} & 1.84958 \times 10^{-18} & -1.85181 \times 10^{-10} \\
3.06163 \times 10^{-3} & -1.85181 \times 10^{-10} & 1.85443 \times 10^{-2}
\end{array}\right]
$$

and the $95 \%$ confidence intervals for the model parameters are given by $b \in[0.4013 \pm 0.0845], \delta \in\left[5.5278 \times 10^{7} \pm 2.6656 \times 10^{-9}\right]$ and $c \in[10.4800 \pm 0.2666]$.

Table 6. Parameter estimates and goodness of fit statistics for various models fitted for silicon nitride data set

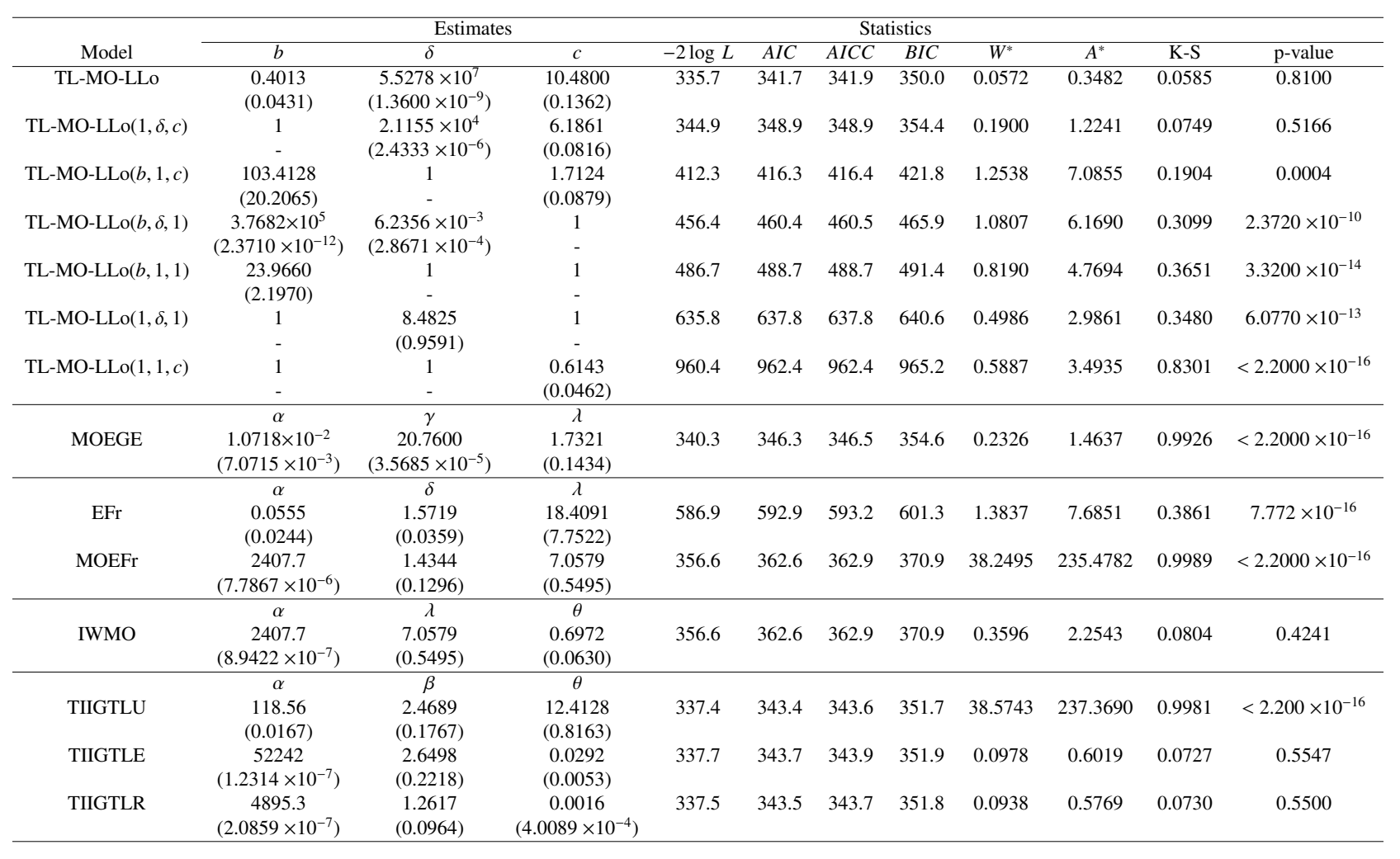

Furthermore, we conclude from the results shown in Table 6 that the TL-MO-LLo distribution fit the silicon nitride data 

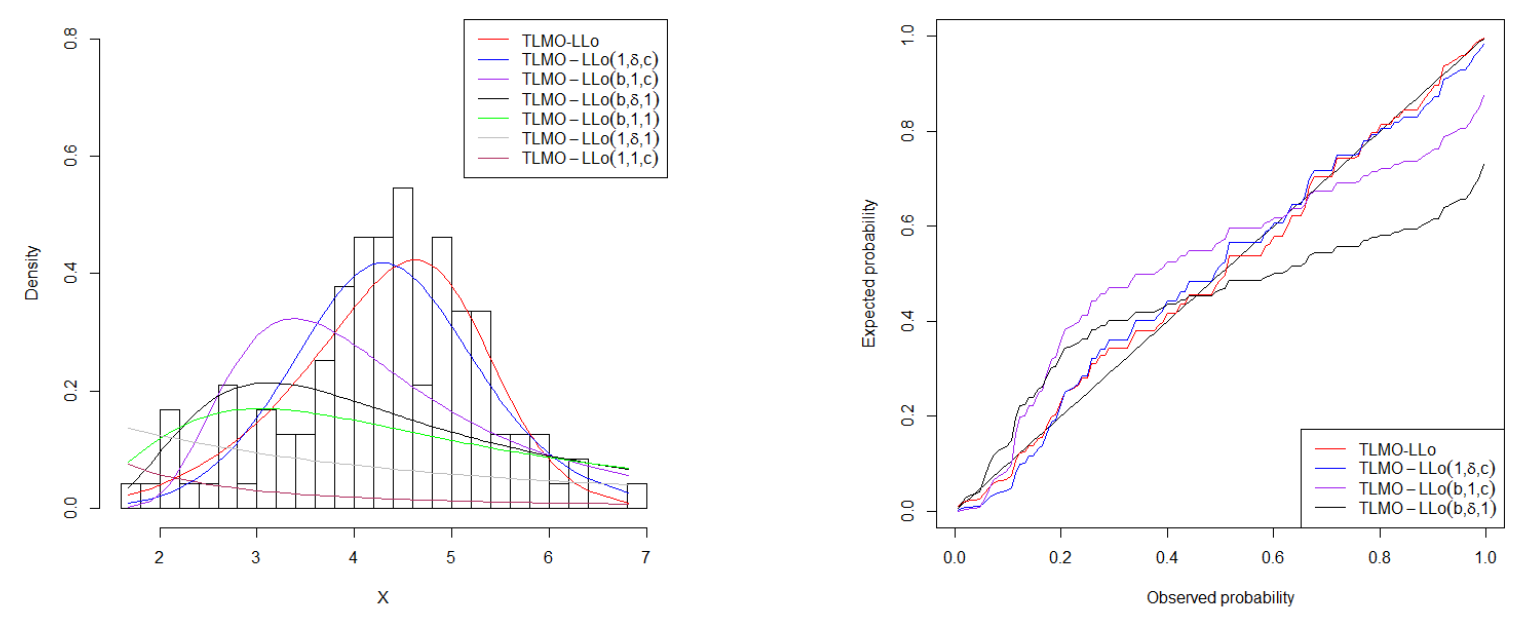

Figure 7. Fitted pdfs and probability plots for silicon nitride data set

set better than the non-nested models considered. The TL-MO-LLo distribution has smaller values of the the goodnessof-fit statistics and a bigger p-value for the K-S statistic. Furthermore, from the fitted densities plots (Figure 7), we can notice the improvement achieved by using the TL-MO-LLo distribution in fitting the silicon nitride data compared to the sub-models.

\subsection{Likelihood Ratio Test}

Likelihood ratio test results for testing if the TL-MO-LLo model performs better than its sub-models models are shown in Table 7.

Table 7. Likelihood Ratio Test Results

\begin{tabular}{lccc}
\hline & Epoxy Kevlar Data & Glass Fibres Data & Silicon Nitride Data \\
Model & $\chi 2(\mathrm{p}$-value $)$ & $\chi 2(\mathrm{p}$-value $)$ & $\chi 2(\mathrm{p}$-value $)$ \\
\hline TLMO-LLo $(1, \delta, c)$ & $11.9(0.000561)$ & $11.2(0.000818)$ & $9.2(0.002420)$ \\
TLMO-LLo $(b, 1, c)$ & $17.0(0.000037)$ & $45.6(<0.00001)$ & $76.6(<0.00001)$ \\
TLMO-LLo $(b, \delta, 1)$ & $14.1(0.000173)$ & $83.9(<0.00001)$ & $120.7(<0.00001)$ \\
TLMO-LLo $(b, 1,1)$ & $17.0(0.000203)$ & $123.0(<0.00001)$ & $151.0(<0.00001)$ \\
TLMO-LLo $(1, \delta, 1)$ & $14.0(0.000912)$ & $178.4(<0.00001)$ & $300.1(<0.00001)$ \\
TLMO-LLo $(1,1, c)$ & $19.4(0.000061)$ & $196.0(<0.00001)$ & $624.7(<0.00001)$ \\
\hline
\end{tabular}

The results from the likelihood ratio test shows that the TL-MO-LLo model performs better than its nested models on all the three data sets.

\section{Concluding Remarks}

We developed a new family of distributions, by combining the Topp-Leone and the Marshall-Olkin-G distributions. The new distribution can handle heavy tailed data and also have non-monotonic hazard rate shapes. The proposed distribution is a linear combination of the Exp-G distribution. We applied the new distribution to three real data sets and our model perform better than the competing non-nested models as shown in Tables 4, 5 and 6.

\section{Acknowledgments}

We are grateful to the referees and the editor for their comments which greatly improved this paper.

\section{References}

Andrews, D. F., \& Herzberg, A. M. (2012). Data: A collection of problems from many fields for the student and research worker. Springer Science \& Business Media.

Ali, A., Hasnain, S. A., \& Ahmad, M. (2015). Modified Burr XII distribution, properties and applications, Pakistan 
Journal of Statistics, 31(6), 697-708.

Alzaatreh, A., Lee, C., \& Famoye, F. (2013). A new method for generating families of continuous distributions. Metron, 71, 63-79. https://doi.org/10.1007/s40300-013-0007-y

Alzaatreh, A., \& Ghosh, I. (2015). On the Weibull-X family of distributions. Journal of Statistical Theory and Applications, 14(2), 169-163. https://doi.org/10.2991/jsta.2015.14.2.5

Barlow, R. E., Toland, R. H., \& Freeman, T. (1984). A Bayesian analysis of stress-rupture life of Kevlar/Epoxy spherical pressure vessels. Proceedings of the Canadian Conference in Applied Statistics, Edited by: Dwivedi, T. D. New York: Marcel Dekker.

Barreto-Souza, W., Lemonte, A. J., \& Cordeiro, G. M. (2013). General results for the Marshall and Olkin's family of distributions. Annals of the Brazilian Academy of Sciences, 85(1), 3-21. https://doi.org/10.1590/S000137652013000100002

Brito, E., Cordeiro, G. M., Yousof, H. M., Alizadeh, M., \& Silva, G. O. (2017). The ToppLeone odd log-logistic family of distributions, Journal of Statistical Computation and Simulation, 87(15), 3040-3058. https://doi.org/10.1080/00949655.2017.1351972

Bourguignon, M., Silva, R. B., \& Cordeiro, G. M. (2014). The Weibull-G family of probability distributions. Journal of Data Science, 12, 53-68.

Chambers, J., Cleveland, W., Kleiner, B., \& Tukey, P. (1983). Graphical methods of data analysis, Chapman and Hall.

Chen, G., \& Balakrishnan, N. (1985). A general purpose approximate goodness-of-fit test. Journal of Quality Technology, 27(2), 154-161. https://doi.org/10.1080/00224065.1995.11979578

Chipepa, F., Oluyede, B., Makubate, B., \& Fagbamigbe A. F. (2019). The beta odd Lindley-G family of distributions with applications. Journal of Probability and Statistical Science, 17(1), 51-83. https://doi.org/10.5539/ijsp.v8n6p1

Chipepa, F., Oluyede, B., \& Makubate, B. (2019). A new generalized family of odd Lindley-G distributions with application. International Journal of Statistics and Probability, 8(6).

Cordeiro, G. M., \& de Castro, M. (2011). A new family of generalized distributions. Journal of Statistical Computation and Simulation, 81(7), 883-898. https://doi.org/10.1080/00949650903530745

Eugene, N., Lee, C., \& Famoye, F. (2002). Beta-normal Distribution and its Applications. Communications in StatisticsTheory and Methods, 31, 497-512. https://doi.org/10.1081/STA-120003130

Hassan, A. S., Elgarhy, M., \& Zubair, A. (2019). Type II generalized Topp-Leone family of distributions: properties and applications. Journal of Data Science, 17(4), 638-659.

Marshall A. N., \& Olkin, I. (1997). A new method for adding a parameter to a family of distributions with applications to the exponential and Weibull families. Biometrika, 84, 641-652. https://doi.org/10.1093/biomet/84.3.641

Nadarajah, S., \& Kotz, S. (2007). On the alternative to Weibull function, Engineering Fracture Mechanics, 74(3), 451456. https://doi.org/10.1016/j.engfracmech.2006.06.007

Nadarajah S., \& Kotz, S. (2003). The exponentiated Fréchet distribution.

Pakungwati, R. M., Widyaningsih, Y., \& Lestari, D. (2018). Marshall-Olkin extended inverse Weibull distribution and its application, Journal of Physics. https://doi.org/10.1088/1742-6596/1108/1/012114

Rényi, A. (1960). On measures of entropy and information, Proceedings of the Fourth Berkeley Symposium on Mathematical Statistics and Probability, 547-561.

Shannon, C. E. (1951). Prediction and entropy of printed english. The Bell System Technical Journal, $30,50-64$. https://doi.org/10.1002/j.1538-7305.1951.tb01366.x

Smith, R. L., \& Naylor, J. C. (1987). A Comparison of maximum likelihood and Bayesian estimators for the threeparameter Weibull distribution. Applied Statistics, 36, 358-369. https://doi.org/10.2307/2347795

Topp, C. W., \& Leone, F. C. (1955). A family of J-shaped frequency functions. Journal of American Statistical Association, 150, 209-219. https://doi.org/10.1080/01621459.1955.10501259

Zografos, K., \& Balakrishnan, N. (2009). On families of beta- and generalized gamma-generated distributions and associated inference. Statistical Methodology, 6, 344-362. https://doi.org/10.1016/j.stamet.2008.12.003 


\section{Copyrights}

Copyright for this article is retained by the author(s), with first publication rights granted to the journal.

This is an open-access article distributed under the terms and conditions of the Creative Commons Attribution license (http://creativecommons.org/licenses/by/4.0/). 